\title{
Rest-frame ultraviolet spectrum of the gravitationally lensed galaxy "the 8 o'clock arc": stellar and interstellar medium properties ${ }^{\star}$
}

\author{
M. Dessauges-Zavadsky ${ }^{1}$, S. D’Odorico ${ }^{2}$, D. Schaerer ${ }^{1,3}$, A. Modigliani ${ }^{2}$, C. Tapken ${ }^{4,5}$, and J. Vernet ${ }^{2}$ \\ 1 Observatoire de Genève, Université de Genève, 51 Ch. des Maillettes, 1290 Sauverny, Switzerland \\ e-mail: miroslava.dessauges@unige.ch \\ 2 European Southern Observatory, Karl-Schwarzschildstr. 2, 85748 Garching bei München, Germany \\ 3 Laboratoire d'Astrophysique de Toulouse-Tarbes, Université de Toulouse, CNRS, 14 Avenue E. Belin, 31400 Toulouse, France \\ 4 Max-Planck-Institut für Astronomie, Königstuhl 17, 69117 Heidelberg, Germany \\ 5 Astrophysikalisches Institut Potsdam, An der Sternwarte 16, 14482 Potsdam, Germany
}

Received 22 September 2009 / Accepted 18 December 2009

\begin{abstract}
We present the first detailed analysis of the rest-frame ultraviolet spectrum of the gravitationally lensed Lyman break galaxy (LBG), the "8 o' clock arc", obtained with the intermediate-resolution X-shooter spectrograph recently commissioned on the ESO Very Large Telescope. Besides MS 1512-cB58, the Cosmic Horseshoe, and the Cosmic Eye, three other lensed LBGs at comparable redshifts, this is the fourth of such a study, usually unfeasible at high redshifts. The spectrum of the 8 o'clock arc is rich in stellar and interstellar features, and presents several similarities to the well-known MS 1512-cB58 LBG. The stellar photospheric absorption lines allowed us to constrain the systemic redshift, $z_{\mathrm{sys}}=2.7350 \pm 0.0003$, of the galaxy, and derive its stellar metallicity, $Z=0.82 Z_{\odot}$, which is in excellent agreement with the metallicity determined from nebular emission lines. With a total stellar mass of $\sim 4.2 \times 10^{11} M_{\odot}$, the 8 o'clock arc agrees with the mass-metallicity relation found for $z>2$ star-forming galaxies, although being located near the upper end of the distribution given its high mass and high metallicity. Broad He II $\lambda 1640$ emission is found, indicative of the presence of Wolf-Rayet stars formed in an intense period of star formation. The 31 interstellar absorption lines detected led to the abundance measurements of 9 elements. The metallicity of the interstellar medium (ISM), $Z=0.65 Z_{\odot}(\mathrm{Si}$ ), is very comparable to the metallicity of stars and ionized gas, and suggests that the ISM of the 8 o'clock arc has been rapidly polluted and enriched by ejecta of OB stars. The ISM lines extend over a very large velocity range, $\Delta v \sim 1000 \mathrm{~km} \mathrm{~s}^{-1}$, from about -800 to $+300 \mathrm{~km} \mathrm{~s}^{-1}$ relative to the systemic redshift, and have their peak optical depth blueshifted relative to the stars, implying gas outflows of $v_{\text {ISM }} \simeq-120 \mathrm{~km} \mathrm{~s}^{-1}$. The zero residual intensity in the strongest lines indicates a nearly complete coverage of the UV continuum by the ISM. The Ly $\alpha$ line is dominated by a damped absorption profile on top of which is superposed a weak emission, redshifted relative to the ISM lines by about $+690 \mathrm{~km} \mathrm{~s}^{-1}$ and resulting from multiply backscattered Ly $\alpha$ photons emitted in the H II region surrounded by the cold, expanding ISM shell. A homogeneous spherical shell model with a constant outflow velocity, determined by the observations, is able to reproduce the observed $\operatorname{Ly} \alpha$ line profile. Furthermore, the required dust content, $E(B-V) \approx 0.3$, is in good agreement with the attenuation measured from the Balmer decrement. These results obtained from the radiation transfer modeling of the Ly $\alpha$ line in the 8 o'clock arc fully support the scenario proposed earlier, where the diversity of Ly $\alpha$ line profiles in Lyman break galaxies and Ly $\alpha$ emitters, from absorption to emission, is mostly due to variations of $\mathrm{H} \mathrm{I}$ column density and dust content.
\end{abstract}

Key words. cosmology: observations - galaxies: individual: 8 o'clock arc - galaxies: starburst - galaxies: abundances

\section{Introduction}

In the quest for high-redshift galaxies and their properties in the early Universe, the most powerful optical, radio, and space telescopes combined with efficient instruments are employed to collect the tiny light emission of these very faint targets. One of the classes of high-redshift galaxies which has been studied in more details is the class of the so-called "Lyman break galaxies".

Lyman break galaxies (LBGs) are UV-selected galaxies characterized by a break in their ultraviolet continuum, that is due to the Lyman limit from intergalactic and interstellar (within the galaxy) HI absorption below $912 \AA$. They are thus easily found using the color-color technique (Steidel et al. 1996). This technique is particularly efficient at $z>2$, where the absorption from the intergalactic medium (IGM) is more pronounced and

* Based on X-shooter observations made with the European Southern Observatory VLT/Melipal telescope, Paranal, Chile, collected during the first $\mathrm{X}$-shooter Commissioning run. the galaxy UV flux is redshifted to optical, allowing observations from the ground. Thousands of LBGs have now been discovered, they are the most common galaxy population detected at $z \sim 2-3$. While many of the global properties of these galaxies, such as their luminosity function, clustering, large-scale distribution, and contribution to the star formation rate density of the Universe, are in the process of being well understood (e.g., Giavalisco et al. 1998; Adelberger et al. 2003; Erb et al. 2006a; Law et al. 2007; Reddy et al. 2008), there have been few detailed spectroscopic studies to date on their individual properties, such as their stellar populations, and chemical enrichment and kinematics of their interstellar medium (ISM).

The limited access to individual properties of LBGs is the result of the faintness of these $L^{*}$ galaxies: their apparent optical magnitudes fainter than $R \simeq 24.5$ at $z \sim 3$ make spectroscopic observations challenging. The high-resolution spectroscopy, in particular, appears as hardly achievable until the $30 \mathrm{~m}$-class telescopes will come into operation. Nevertheless, 
rest-frame UV and optical, low-resolution composite spectra of LBGs have begun to provide some insights into the physical properties of these high-redshift galaxies. It appears that LBGs resemble present-day star-forming galaxies with spectra characterized by young and massive stellar populations of nearsolar metallicities, dominated by on-going star formation, with strong outflows, and dusty component within abundant neutral gas (e.g., Rix et al. 2004; Shapley et al. 2003, 2004; Erb et al. 2006b).

For a few objects, nature provides an alternative route to bypass the step of next generation telescopes with large collecting areas and study individual LBGs spectroscopically at medium and high resolutions. This happens in case of fortuitous alignments of LBGs with foreground mass concentrations which lead to light magnification due to gravitational lensing. The bestknown example is the LBG MS 1512-cB58 (cB58) that is exceptionally bright for its redshift $z=2.73$, benefiting of a lensing magnification factor of $\sim 30(g=21.08, r=20.60$; Ellingson et al. 1996). This allowed a uniquely detailed chemical and kinematical analysis of the ISM, stars, H II regions, Ly $\alpha$ profile, and surrounding IGM of this high-redshift galaxy (Pettini et al. 2000, 2002; Teplitz et al. 2000; Savaglio et al. 2002; Rix et al. 2004; Schaerer \& Verhamme 2008).

Recently, new search techniques for strongly-lensed highredshift galaxies, mainly based on the Sloan Digital Sky Survey (SDSS), have yielded additional LBG candidates (see e.g., Kubo et al. 2009, and references therein). All these objects are excellent targets for follow-up observations with intermediate-tohigh resolution spectrographs in the optical and/or in the nearinfrared (NIR). So far, Quider et al. (2009a) provided a first similar in-depth study as the one of cB58 of the lensed LBG the "Cosmic Horseshoe" at $z=2.38$ (J1148+1930; Belokurov et al. 2007), thanks to rest-frame UV spectra obtained at intermediateresolution with the ESI/Keck II spectrograph. And similarly, Quider et al. (2009b) published the detailed analysis of the restframe UV spectra of the "Cosmic Eye", a lensed LBG at $z=3.07$ (J213512.73-010143; Smail et al. 2007). Hainline et al. (2009), on the other hand, led a very detailed study of the rest-frame optical spectra of three lensed LBGs - the Cosmic Horseshoe, the Clone at $z=2.00$ (Lin et al. 2009), and SDSS J0901+1814 at $z=2.26$ (Diehl et al. 2009) - obtained at moderate-resolution with the NIRSPEC/Keck II spectrograph. Finally, Cabanac et al. (2008) undertook a detailed analysis of the rest-frame UV spectra of the lensed LBG FOR J0332-3557 at a higher redshift $z=$ 3.77 (Cabanac et al. 2005), acquired with the FORS2/VLT spectrograph, at a resolution about 4 times lower than that of $\mathrm{cB58}$, Cosmic Horseshoe, and Cosmic Eye spectra.

The lensed Lyman break galaxy the "8 o" clock arc", discovered by Allam et al. (2007) in the SDSS Data Release 4, is another very exciting example of high-redshift galaxies which individual physical properties can be studied in great details. Indeed, the 8 o'clock arc at $z \simeq 2.73$ is even the brightest LBG currently known, with an apparent brightness 3 times higher than the one of cB58 $(g=19.95, r=19.22)$. It is strongly lensed by the $z=$ 0.38 luminous red galaxy (LRG) SDSS J002240.91+143110.4, resulting in a total magnification factor $\mu=12.3_{-3.6}^{+15}$. Even after accounting for this magnification, the 8 o' clock arc is intrinsically more luminous by about $2.6 \mathrm{mag}$ (a factor of $\sim 11$ ) than typical $L^{*}$ LBGs. The lensing distorts the galaxy into four separate knots. Three of them A1-A3 form a partial Einstein ring of radius $\theta_{\mathrm{E}}=3.32^{\prime \prime} \pm 0.16^{\prime \prime}$, subtending an angle of $162^{\circ}$ and extending over 9.6" in length (see Fig. 1). Finkelstein et al. (2009) reported the first study of low-resolution rest-frame UV and optical spectra of this newly discovered LBG obtained with

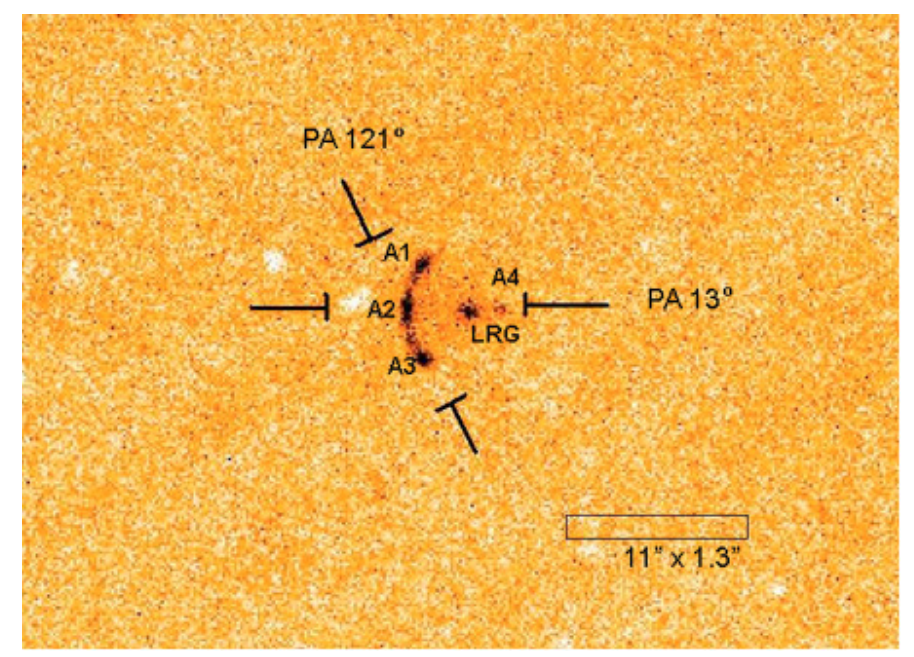

Fig. 1. $45 \mathrm{~s}$ X-shooter acquisition image of the 8 o'clock arc obtained through the $g^{\prime}$ SDSS filter. The two slit orientations selected for the 8 o'clock arc observations are shown (see Table 1). The various lens images are labeled according to Allam et al. (2007). The size of the slit we used for the observations with the UV-B spectrograph is also plotted. In this good seeing $\left(0.6^{\prime \prime}\right)$ image, emission along the whole arc in between the main knots A1-A3 - is clearly visible. Diffuse emission is also seen all around the lens galaxy and, in particular, in the region having the arc as the outer boundary.

the LRIS/Keck I and NIRI/Gemini North spectrographs, respectively. They derived several important physical quantities, such as a metallicity of $\sim 0.8 Z_{\odot}$ from $\mathrm{H}$ II regions, a dust extinction of $A_{5500}=1.17 \pm 0.36 \mathrm{mag}$, a stellar mass of $\sim 4.2 \times 10^{11} M_{\odot}$, and a star formation rate of $\sim 270 M_{\odot} \mathrm{yr}^{-1}$.

The 8 o'clock arc was chosen as a target for the first Commissioning period of the new X-shooter spectrograph on the Very Large Telescope (VLT). High-quality rest-frame UV spectra were acquired of this lensed LBG at intermediateresolution. These spectra are complementary to those acquired by Finkelstein et al. (2009). Besides MS 1512-cB58, the Cosmic Horseshoe, and the Cosmic Eye, with the 8 o'clock arc we provide the fourth unprecedented detailed study of stars and ISM gas, from the rest-frame UV spectrum analysis, of a highredshift Lyman break galaxy. The build-up of a sample of such comprehensive studies is necessary to better understand the physical properties of these high-redshift galaxies and to determine how typical is the well-know case of cB58.

The layout of the paper is as follows. In Sect. 2 we report on properties of the new X-shooter instrument, on observations, and on data reduction procedures. In Sect. 3 we discuss the stellar spectrum of the galaxy, and determine the systemic redshift and the metallicity of OB stars. In Sect. 4 we present the interstellar spectrum, and derive the ion column densities. In Sect. 5 we analyze and model the Ly $\alpha$ line profile. Finally, in Sect. 6 we summarize the results and discuss them in the context of properties of other Lyman break galaxies. Throughout the paper, we adopt the standard cosmology with $H_{0}=70 \mathrm{~km}^{-1} \mathrm{Mpc}^{-1}, \Omega_{\mathrm{M}}=0.3$, and $\Omega_{\Lambda}=0.7$.

\section{Observations and data reduction}

$\mathrm{X}$-shooter is the first of the 2nd generation VLT instruments (D'Odorico et al. 2006) currently mounted on the $8.2 \mathrm{~m}$ Kueyen telescope at Cerro Paranal, Chile. It was built by a consortium of institutes in Denmark, France, Italy, and The Netherlands, 
Table 1. X-shooter observations of the 8 o'clock arc.

\begin{tabular}{lllccl}
\hline \hline & Observing date & Arm & $\begin{array}{c}\text { Resolution } \\
R=\lambda / \Delta \lambda\end{array}$ & $\begin{array}{c}\text { Exposure time } \\
(\mathrm{s})\end{array}$ & Slit position $^{a}$ \\
\hline$\# 1$ & Nov. 16, 2008 & UV-B & 4000 & 3600 & PA $=118^{\circ}$ along A2 and A3 \\
$\# 2$ & Nov. 16, 2008 & VIS-R & 6700 & 3600 & Same as \#1 \\
$\# 3$ & Nov. 16, 2008 & UV-B & 4000 & 3600 & PA $=118^{\circ}$ along A2 and A3 \\
$\# 4$ & Nov. 16, 2008 & VIS-R & 6700 & 3600 & Same as \#3 \\
$\# 5$ & Nov. 18, 2008 & UV-B & 4000 & 4500 & PA $=121^{\circ}$ along A2 and A3 \\
$\# 6$ & Nov. 18, 2008 & VIS-R & 6700 & 4500 & Same as \#5 \\
$\# 7$ & Nov. 19, 2008 & UV-B & 4000 & 4500 & PA $=13^{\circ}$ along A2 and the LRG \\
$\# 8$ & Nov. 19, 2008 & VIS-R & 6700 & 4500 & Same as \#7 \\
\hline
\end{tabular}

Notes. ${ }^{(a)}$ Labels of the lens images as in Allam et al. (2007) and Fig. 1.

and by the European Southern Observatory (ESO) which was responsible for its final integration and installation at the telescope. It consists of three echelle spectrographs with prism crossdispersion mounted on a common structure at the Cassegrain focus. The light beam from the telescope is split in the instrument by two dichroics which direct the light in the spectral ranges of $300-560 \mathrm{~nm}$ and $560-1015 \mathrm{~nm}$ to the slit of the ultraviolet-blue (UV-B) and visual-red (VIS-R) spectrographs, respectively. The undeviated beam in the spectral range of 1025-2400 nm feeds the near-infrared (NIR) spectrograph. The UV-B and VIS-R spectrographs operate at ambient temperature and pressure and deliver $2 \mathrm{D}$ spectra on $4 \mathrm{k} \times 2 \mathrm{k}$ pixels CCDs. The NIR spectrograph is enclosed in a vacuum vessel and kept at a temperature of $\sim 80 \mathrm{~K}$ by a continuous flow of liquid nitrogen. The spectral format in the three spectrographs is fixed, with the possibility to change the resolution by using slits of different widths.

After two Commissioning runs with the UV-B and VIS-R spectrographs in November 2008 and January 2009, the instrument is operating in its full configuration, including the NIR spectrograph, from March 2009. Calibrations and data of scientific value obtained during the Commissioning runs are part of the public release by the ESO archive ${ }^{1}$. The data used in this paper were obtained during the first Commissioning run of November 2008. These were the first nights of the instrument at the telescope, when the Commissioning team was testing the observing procedures and the instrument behavior on sky targets. The 8 o'clock arc was considered as a good test-case of faint galaxy observations. While the instrument set-up, the observing strategy, and the exposure times were not optimized yet, the acquired spectra are still mostly of good quality and provide unique data of the 8 o'clock Lyman Break galaxy.

The list of observations is summarized in Table 1. A total exposure time of $16200 \mathrm{~s}$ ( 2 exposures of $3600 \mathrm{~s}$ and 2 others of $4500 \mathrm{~s}$ ) was obtained on the 8 o'clock arc in good conditions, with a clear sky and seeing between $0.6^{\prime \prime}$ and $1.2^{\prime \prime}$. Slit widths of $1.3^{\prime \prime}$ in the UV-B and $1.2^{\prime \prime}$ in the VIS-R were used, corresponding to resolutions $R=4000$ and $R=6700$, respectively. Two main slit orientations were selected aligned at position angles $\mathrm{PA} \simeq 121^{\circ}$ and $13^{\circ}$, along the 8 o' clock knots A2 and A3 and along the knot A2 and the lens galaxy, respectively. Figure 1 shows the two slit positions on the sky on a $45 \mathrm{~s}$ acquisition image obtained with X-shooter through the $g^{\prime}$ SDSS filter. All the observations were conducted at air mass of $\sim 1.3$.

\footnotetext{
1 http://www.eso.org/sci/facilities/develop/ instruments/xshooter/X-shooter_CommDataRelease_text. html
}

The UV-B exposures \#1, \#3 and \#5 show an in-focus light ghost which at wavelengths below $380 \mathrm{~nm}$ partly overlaps the signal of the 8 o'clock knots A2 and A3, and below $460 \mathrm{~nm}$ prevents an accurate sky subtraction in exposures \#3 and \#5. After excluding possible contamination from some parasitic light sources in the instrument or the telescope, the bright lensing LRG, at less than 3 arcsec off the slit, was suspected as the source of the ghost. This is supported by the exposure \#7, taken with the slit aligned along the knot A2 and the LRG, which does not show any light ghost. However, the effect was not observed with any other target, and various tests made with bright stars close to the slit failed to reproduce it. The matter is still under investigation by the instrument team.

The X-shooter spectra were reduced with the Beta version of the X-shooter reduction pipeline (Goldoni et al. 2006) running at ESO. Pixels in the 2D echelle format frames are mapped in the wavelength space using calibration frames. Sky emission lines are normally subtracted before any resampling, using the method developed by Kelson (2003). The different orders are then extracted, rectified, wavelength calibrated with a constant spectral bin, and merged with a weighted average used in the overlapping regions.

Given the complexity of the geometry of the 8 o'clock arc target and the related observations where one single slit is aligned along two knots, we had to perform the object extraction and sky subtraction manually. For this purpose, we used the 2D wavelength calibrated, resampled, background subtracted, order merged UV-B and VIS-R spectra as produced by the pipeline, and made manual object and sky extractions by carefully selecting the respective extraction windows. The separate extraction of the knots A2 and A3 in exposures \#1 to \#6 led to very low signal-to-noise ratio $(\mathrm{S} / \mathrm{N})$ individual spectra. Since no difference is observed between the A2 and A3 spectra within the limits of the noise and the signal of the two knots in the slit is partly blended, we handled the sum of the signals of the two knots. In the case of exposures \#7 and \#8, where the slit was aligned along the knot A2 and the LRG, the extracted object spectrum corresponds solely to the knot A2. Exposures with this specific slit orientation were extremely useful to estimate possible light contamination of the 8 o'clock arc by the lensing galaxy. Indeed, they show a residual emission relative to the sky signal in between the LRG and the knot A2. The extracted light profile along the $11^{\prime \prime}$ slit from the nucleus of the LRG up to the sky beyond the knot A2 (see Fig. 1) follows the de Vaucouleurs law with the typical $R^{1 / 4}$ intensity profile of elliptical galaxies (where $R$ is the distance relative to the LRG). A significant wavelength dependent contamination by the LRG (increasing toward longer wavelengths), of the order of $\sim 30 \%$, is observed at the position of the 8 o'clock arc. We thus subtracted this light 
Table 2. Stellar photospheric absorption lines and emission lines.

\begin{tabular}{lcllcccc}
\hline \hline Ion & $\begin{array}{c}\lambda_{\text {lab }}{ }^{a} \\
(\AA)\end{array}$ & Origin & $z_{\text {stars }}{ }^{b}$ & $z_{\mathrm{em}^{c}}$ & $\begin{array}{c}\Delta v^{d} \\
\left(\mathrm{~km} \mathrm{~s}^{-1}\right)\end{array}$ & $\begin{array}{c}W_{0}{ }^{e} \\
(\AA)\end{array}$ & $\begin{array}{c}\sigma\left(W_{0}\right)^{e} \\
(\AA)\end{array}$ \\
\hline Si III & 1294.543 & stars & 2.7349 & & -165 to +105 & 0.13 & 0.02 \\
C III & 1296.330 & stars & 2.7349 & & -165 to +195 & $0.16^{f}$ & $0.02^{f}$ \\
Si III & 1296.726 & stars & 2.7348 & & -165 to +195 & $0.16^{f}$ & $0.02^{f}$ \\
C II & 1323.929 & stars & 2.7348 & & -165 to +195 & $0.21^{g}$ & $0.03^{g}$ \\
N III & 1324.316 & stars & 2.7345 & & -165 to +195 & $0.21^{g}$ & $0.03^{g}$ \\
Si II & 1533.431 & recombination & & 2.7352 & -95 to +185 & -0.20 & 0.02 \\
N IV & 1718.551 & stars & 2.7347 & & -210 to +165 & 0.57 & 0.06 \\
[C III] & 1906.683 & H II regions & & 2.7348 & -185 to +165 & -0.35 & 0.04 \\
C III] & 1908.734 & H II regions & & 2.7352 & -165 to +185 & -0.26 & 0.03 \\
\hline
\end{tabular}

Notes. ${ }^{(a)}$ Vacuum wavelengths; ${ }^{(b)}$ vacuum heliocentric and values measured from the centroid wavelength of the photospheric absorption lines; ${ }^{(c)}$ vacuum heliocentric and values measured from the Gaussian fits to the emission lines; ${ }^{(d)}$ velocity range for equivalent width measurements relative to $z_{\text {sys }}=2.7350 ;{ }^{(e)}$ rest-frame equivalent width and $1 \sigma$ error; ${ }^{(f)}$ this value refers to the blend of lines $\mathrm{C}$ III $\lambda 1296.330$ and $\mathrm{Si}$ III $\lambda 1296.726$; (g) this value refers to the blend of lines C II $\lambda 1323.929$ and N III $\lambda 1324.316$.

contamination in addition to the manually extracted sky signal (as determined at larger distances from the lens) from the extracted signals of the knots A2 and A3 in all exposures. The 1D science spectra from the different exposures were then coadded using their $\mathrm{S} / \mathrm{N}$ as weights ${ }^{2}$. For this, the wavelength scale was first converted to the vacuum-heliocentric scale. The spectra were finally normalized by smoothly connecting regions free from absorption features with a spline function. A particularly careful continuum fitting was done in the Ly $\alpha$ forest.

The final UV-B and VIS-R spectra have, respectively, a S/N per resolution element of $\sim 10$ from 440 to $560 \mathrm{~nm}$ and $\sim 13$ from 560 to $800 \mathrm{~nm}$. From the widths of the sky emission lines, we measured a spectral resolution of $1.25 \AA\left(69 \mathrm{~km} \mathrm{~s}^{-1}\right) F W H M$ at $540 \mathrm{~nm}$ in the UV-B spectrum and $0.85 \AA\left(42 \mathrm{~km} \mathrm{~s}^{-1}\right) F W H M$ at $600 \mathrm{~nm}$ in the VIS-R spectrum. This is in very good agreement with the resolutions expected with the slit widths used for our observations. The sky emission lines allowed us also to check the wavelength calibration of our X-shooter spectra. The achieved wavelength calibration accuracy is $\sim 10 \mathrm{~km} \mathrm{~s}^{-1} \mathrm{rms}$ in both the UV-B and VIS-R spectra.

\section{The stellar spectrum}

The rest-frame UV spectrum of the 8 o'clock arc consists of the integrated light from the hot and luminous $\mathrm{O}$ and $\mathrm{B}$ stars in the galaxy (the stellar spectrum) on which are superposed the resonant absorption lines produced by the interstellar gas (the interstellar spectrum). The careful analysis of all these resulting lines brings precious information on the physical properties of both the stars and gas in this high redshift galaxy. On top of these lines, absorption from the Ly $\alpha$ forest and several intervening metal-line systems is also found. We discuss first the stellar spectrum.

\subsection{Systemic redshift}

Most of the low-contrast/weak structure seen in the continuum of high $\mathrm{S} / \mathrm{N}$ spectra of star-forming galaxies is caused by stellar features, not noise. These features are largely blends of different stellar lines which require stellar population synthesis to be analyzed quantitatively (see Sect. 3.2). Pettini et al. (2000, 2002) identified a few stellar photospheric lines that appear to be least

\footnotetext{
2 Solely exposures \#1 and \#7 were co-added in the UV-B, because sky subtraction failed in exposures \#3 and \#5 due to the presence of the light ghost (see above).
}

affected by blending, and can therefore provide a measure of the systemic redshift of the stellar population.

We identified 6 of these photospheric absorption lines. They are listed in Table 2 with their redshifts and rest-frame equivalent widths, $W_{0}$, and plotted in velocity space in Fig. 2 . The tabulated redshifts ${ }^{3}$ are those derived from the centroid of the lines, that corresponds to the mean wavelength of the line weighted by the absorption at each wavelength. Solely the redshift derived from the NIV $\lambda 1718$ line provides a reliable measure of $z_{\text {stars }}=2.7347 \pm 0.0003$, given the larger strength of this line with respect to the other stellar photospheric absorption lines only marginally detected $(2-4 \sigma)$ and the blends of C III with Si III and C II with N III.

Also included in Table 2 are 3 emission lines: the nebular C III] $\lambda \lambda 1906,1908$ doublet clearly resolved in our $\mathrm{X}$-shooter spectrum of the 8 o'clock arc and the fine-structure Si II* $\lambda 1533$ emission line (Fig. 2). The mean $z_{\mathrm{em}}=2.7351 \pm$ 0.0002 is in very good agreement with $z_{\text {stars }}$, differing by only $32 \mathrm{~km} \mathrm{~s}^{-1}$. We thus adopt throughout the paper $z_{\text {sys }}=2.7350 \pm$ 0.0003 - the mean of $z_{\mathrm{stars}}$ and $z_{\mathrm{em}}-$ as the systemic redshift in the 8 o'clock arc.

Finkelstein et al. (2009) measured $z_{\mathrm{H} \text { II }}=2.7333 \pm 0.0001$ from the mean of 6 well-detected emission lines formed in $\mathrm{H}$ II regions redshifted into the NIR. The large difference, $\Delta v=$ $-136 \mathrm{~km} \mathrm{~s}^{-1}$, between $z_{\mathrm{H} \text { II }}$ and $z_{\text {sys }}$ derived here (based also on two nebular C III] emission lines) is difficult to explain. Moreover, their redshift estimates from the stellar photospheric and interstellar lines redshifted into the optical show the same discrepancy. The higher resolution and wavelength accuracy of our X-shooter spectra than of their observations lead us to preferentially trust our redshift estimates.

\subsection{Photospheric absorption lines}

Similarly to Pettini et al. (2000), Cabanac et al. (2008), and more recently to Quider et al. (2009a,b) who performed the stellar modeling of the integrated spectra of four lensed LBGs, respectively, we analyze the photospheric and wind lines in the spectrum of the 8 o'clock arc. For this, we compare our data with the synthetic spectra computed by Rix et al. (2004) using their updated version of the population synthesis code Starburst 99 (Leitherer et al. 1999, 2001) which couples libraries of theoretical UV OB stellar spectra with stellar evolutionary tracks. We follow their assumption of a continuous star formation mode -

\footnotetext{
${ }^{3}$ All redshifts quoted in this paper are vacuum heliocentric.
} 


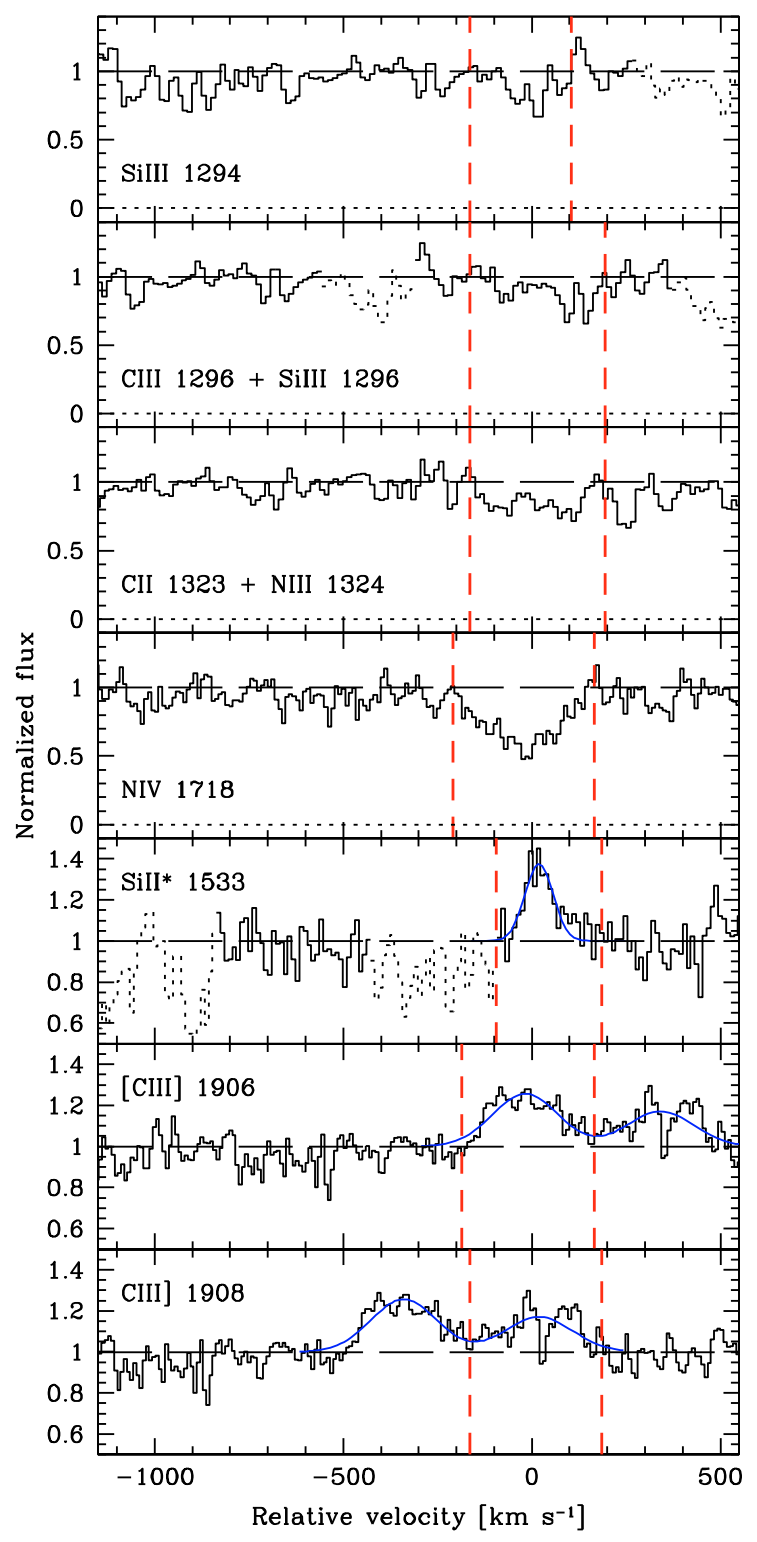

Fig. 2. Velocity plots of normalized profiles of the stellar photospheric absorption lines and the emission lines detected in the 8 o'clock arc. The zero velocity is fixed to $z_{\text {sys }}=2.7350$. The vertical red dashed lines indicate the velocity range over which the equivalent widths listed in Table 2 are measured. The blue solid lines are Gaussian fits to the emission lines. The dotted lines are used to indicate blends with absorption features that are not those labeled in each panel.

with a Salpeter initial mass function (IMF) between 1 and $100 M_{\odot}$ and a constant star formation rate for $100 \mathrm{Myr}-$ which seems to be the better description for most LBGs.

Leitherer et al. (2001) and Rix et al. (2004) explored in detail a number of spectral regions in the integrated UV stellar spectra of star-forming galaxies that are sensitive to metallicity and clean of other spectral features. They identified three very promising metallicity indicators: (1) the "1370" index which arises from the blending of the $\mathrm{OV} \lambda 1371$ and $\mathrm{FeV} \lambda \lambda 1360$, 1380 absorption lines over $1360-1380 \AA$; (2) the " 1425 " index which arises from the blending of the Si III $\lambda 1417$, C III $\lambda 1427$ and Fe V $\lambda 1430$ absorption lines over 1415-1435 $\AA$; and (3) the "1978" index which arises from the blending of numerous Fe III transitions over 1935-2020 А. The equivalent widths of all these indices increase monotonically with metallicity. The
"1978" index is particularly interesting, because it has a larger equivalent width and is free of contaminating interstellar lines.

To compare our high-resolution X-shooter spectra of the 8 o'clock arc with the synthetic stellar spectra of Rix et al. (2004), we smoothed our non-normalized spectra to the resolution $F W H M=2.5 \AA$ of the synthetic spectra (in the rest-frame wavelength scale) by convolution with Gaussian profiles of the appropriate widths. We then normalized the smoothed X-shooter spectra by division by a spline curve through the mean flux in each of the pseudo-continuum windows deemed to be free of absorption/emission features identified by Rix et al. (2004, Table 3). Given the broad and shallow nature of the photospheric blends making up the "1370", "1425", and "1978" indices, their equivalent widths are very sensitive to the continuum normalization, which in turn depends on the spectral resolution of the spectra. It is therefore mandatory to apply the above steps on the acquired data.

In the panels of Fig. 3 are shown portions of the smoothed $\mathrm{X}$-shooter spectra of the 8 o'clock arc with the synthetic stellar spectra of Rix et al. (2004) for 5 values of metallicity, from 1/20

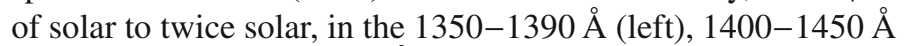
(middle), and 1900-2030 $\AA$ (right) wavelength intervals, corresponding to the "1370", "1425", and "1978" metallicity indices, respectively. The synthetic spectra do a remarkably good job at reproducing the observed spectra; except for the strong absorption between 1400 and $1410 \AA$ which corresponds to the interstellar Si IV $\lambda 1402$ line plus an intervening metal-line and the emission at $\sim 1907 \AA$ which is the blend of the nebular C III] $\lambda \lambda 1906,1908$ doublet, that are unaccounted for in the Rix et al. models. Among the 5 metallicities considered, $Z=1 Z_{\odot}$ and $0.4 Z_{\odot}$ are those that most closely match the observations in all the "1370", "1425", and "1978" regions. Using the relations of Rix et al. (2004) between the equivalent widths of the " 1425 " and " 1978 " line blends and $\log \left(Z / Z_{\odot}\right)$, we get a first estimate of the metallicity of the 8 o' clock LBG: the measured $W_{0}(1425)=1.16 \AA$ over the $1415-1435 \AA$ interval gives $Z=0.85 Z_{\odot}$, and the measured $W_{0}(1978)=5.56 \AA$ over the 1935-2020 $\AA$ interval gives $Z=0.79 Z_{\odot}$. The two metallicities are in excellent agreement.

The P-Cygni lines formed in the expanding winds of the most luminous OB stars are other very important metallicity indicators. The optical depth of these lines is sensitive to the mass-loss rate which in turn decreases with decreasing metallicity. The C IV $\lambda \lambda 1548,1550$ doublet is the strongest P-Cygni line covered by the X-shooter spectra of the 8 o'clock arc. We do not consider the C IV doublet here as a metallicity indicator, as no precise metallicity calibration exists for these lines so far. The stellar models of Rix et al. (2004) are not applicable, because at the resolution $F W H M=2.5 \AA$ of their synthetic spectra the interpretation of the C IV P-Cygni line is complicated by its blending with the interstellar C IV doublet and to some extent the Si II $\lambda 1526$ absorption.

\subsection{Emission lines}

As did Quider et al. (2009a) in the Cosmic Horseshoe, we detect the nebular C III] $\lambda \lambda 1906,1908$ doublet formed in $\mathrm{H}$ II regions that is clearly resolved in our X-shooter spectra of the 8 o'clock arc (see Fig. 2 ). The mean redshift, $z_{\mathrm{C} \text { III] }}=2.7350$, is in very good agreement with both the redshift of the stellar photospheric absorption lines, $z_{\text {stars }}$, and the redshift of another detected emission line, Si II* $\lambda 1533$ (see Table 2). According to Pettini et al. (2000), Si II* can be interpreted as a recombination 


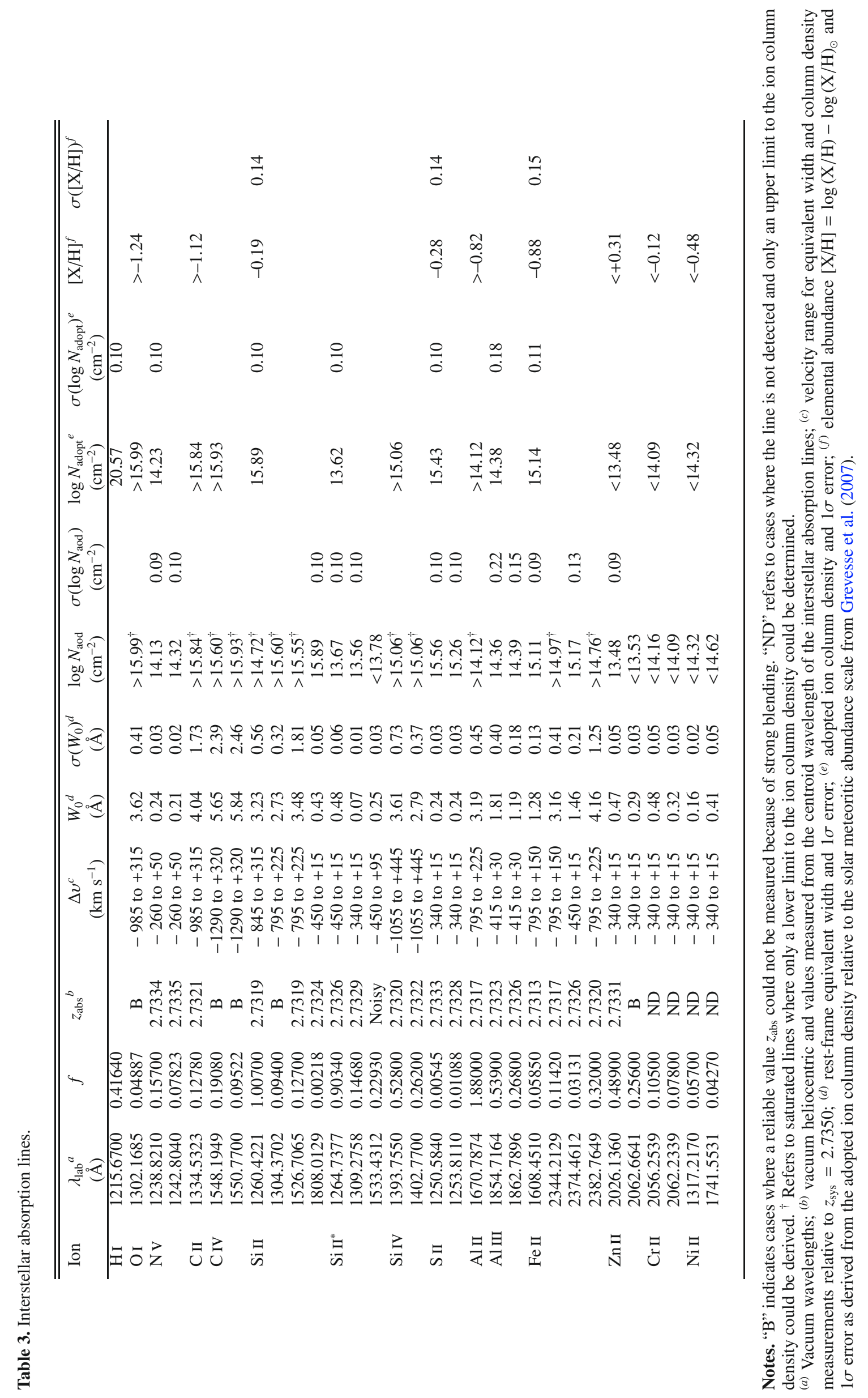



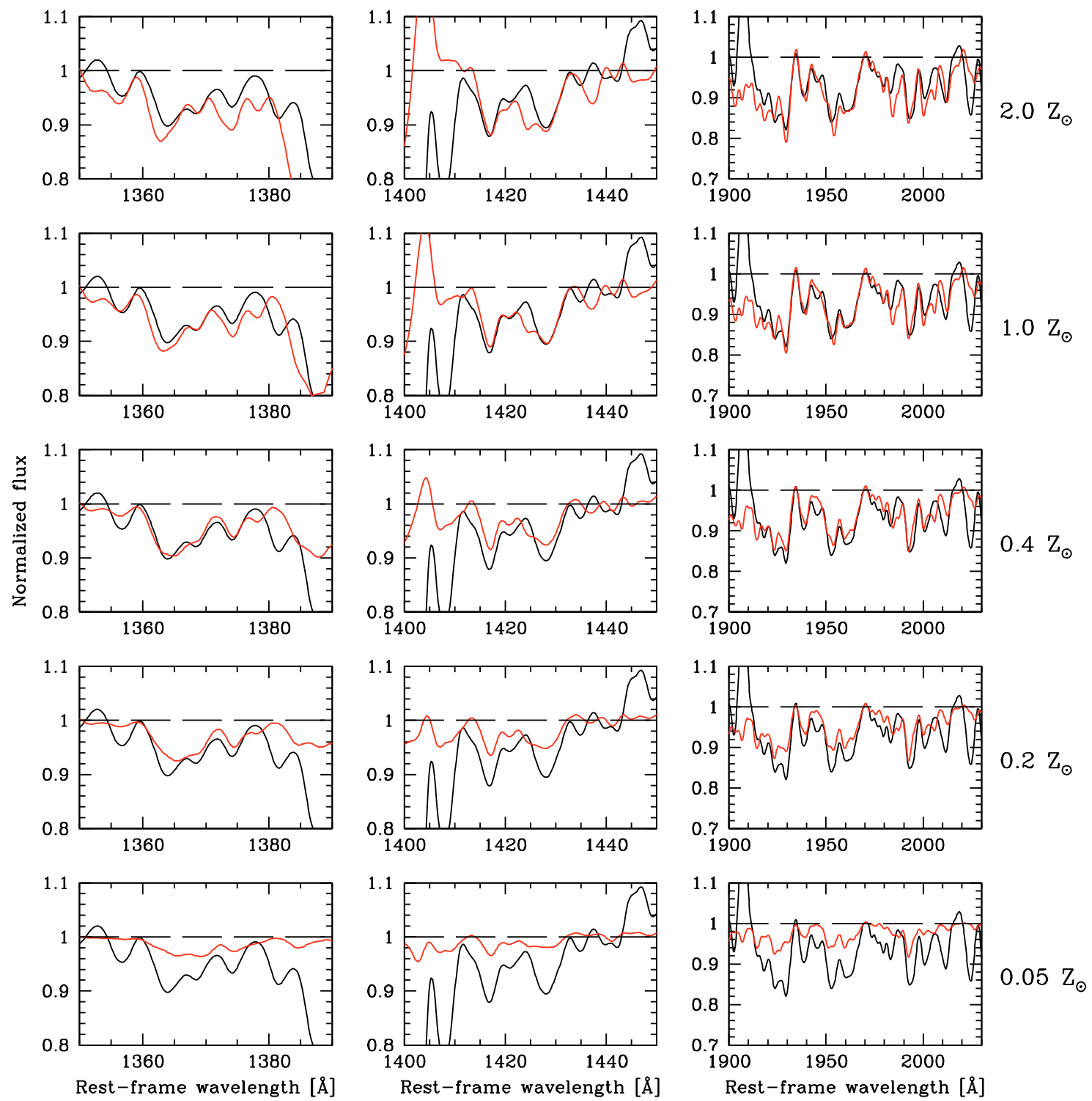

Fig. 3. Comparison of the X-shooter spectra of the 8 o'clock arc smoothed to the $F W H M=2.5 \AA$ resolution (black) with synthetic stellar spectra (red) produced by Rix et al. (2004) for 5 metallicities, from twice solar to 1/20 of solar, as indicated. Wavelength portions encompassing the "1370", "1425", and "1978" metallicity indices are plotted from the left to the right, respectively. The strong absorption between 1400 and $1410 \AA$ corresponds to the interstellar Si IV $\lambda 1402$ line plus an intervening metal-line and the emission at $\sim 1907 \AA$ to the blend of the nebular C III] $\lambda \lambda 1906$, 1908 doublet, that are unaccounted for in the Rix et al. models.

line to the fine-structure level of the ground state of Si II, presumably arising in an $\mathrm{H}$ II region. We also searched for nebular O III] $\lambda \lambda 1660.809,1666.150$ emission, but these lines appear to be below the detection limit of our data.

Applying Gaussian fits to the C III] doublet allows to derive the full width at half maximum, $F W H M$, and hence the velocity dispersion, $\sigma=F W H M / 2.355 \times c / \lambda_{\mathrm{obs}}$, which is a measure of the dynamics of the gas bound to the galaxy by gravity. We obtain very consistent velocity dispersions for the two C III] lines, $\sigma_{\text {CIII] }}=70$ and $71 \mathrm{~km} \mathrm{~s}^{-1}$, respectively (after correcting for the instrumental resolution). The mean value is in very good agreement with the velocity dispersions obtained by Hainline et al. (2009) for the few lensed LBGs known so far, while it is about twice the velocity dispersion obtained by Finkelstein et al. (2009) for the nebular $\mathrm{H} \alpha$ line redshifted into the NIR.

The ratio of the two $\mathrm{C} \mathrm{III]}$ lines is a function of the electron density. It varies from values of about 1.5 to 0.8 in the range of $n(e)=100$ to $30000 \mathrm{~cm}^{-3}$, respectively. The measured C III] $\lambda 1906 / \lambda 1908$ line ratio of $\sim 1.5$ in the 8 o'clock arc points to the lowest end of electron densities, $n(e) \sim 100$. This is consistent with the values usually observed in local star-forming galaxies, but appears lower than the electron densities determined from the nebular [S II] $\lambda \lambda 6717,6731$ doublet in the other highredshift LBGs (Brinchmann et al. 2008; Hainline et al. 2009).

Interestingly, we also detect another very broad emission feature in the 8 o'clock arc, extending over $\sim 75 \AA(F W H M \sim$ $\left.2000 \mathrm{~km} \mathrm{~s}^{-1}\right)$, which at the systemic redshift $z_{\text {sys }}=2.7350$ is identified as the He II $\lambda 1640.418$ line (see Fig. 4). The He II emission is known to be a possible signature of very massive stars, produced by Wolf-Rayet (WR) stars. Hints for this emission were already detected in the composite spectrum of LBGs of Shapley et al. (2003), and more recently Cabanac et al. (2008) reported a first clear detection in the lensed FOR J0332-3557 LBG. 


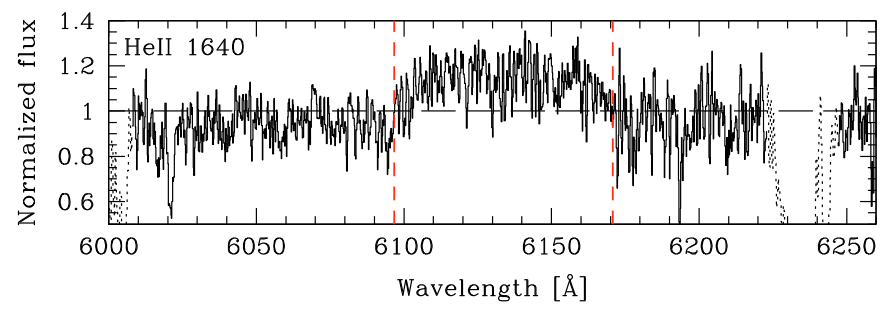

Fig. 4. Normalized profile of the He II $\lambda 1640$ emission line detected in the 8 o'clock arc. The vertical red dashed lines indicate the wavelength range over which the equivalent width is measured. The dotted lines are used to indicate ISM absorption features.

We measure a rest-frame equivalent width $W_{0}(\mathrm{He}$ II $)=$ $-2.45 \pm 0.22 \AA$, very similar to the value obtained by Cabanac et al. (2008) in FOR J0332-3557, but somewhat larger than the value of $W_{0}(\mathrm{He}$ II $)=-1.3 \pm 0.3 \AA$ measured by Brinchmann et al. (2008) in the composite spectrum of $z \sim 3$ LBGs of Shapley et al. (2003). Comparison with local measurements suggests that the equivalent width of He II $\lambda 1640$ in the 8 o' clock arc is similar to values found in nearby starburst super-star clusters (Chandar et al. 2004, Table 3). Comparison with evolutionary synthesis models (Schaerer \& Vacca 1998; Brinchmann et al. 2008) shows that the strength of the observed He II emission can be understood by either relatively young bursts of $\lesssim 10 \mathrm{Myr}$, by continuous star formation at solar metallicity over $\$ 20 \mathrm{Myr}$, or by longer star formation timescales at metallicities above solar. We conclude that the strong He II $\lambda 1640$ emission line in the 8 o'clock arc very likely results from WR stars. However, given the uncertainties involved in the models (e.g., line luminosity calibrations, evolution of massive stars) and in the precise star formation history, we cannot draw more solid conclusions from this spectral feature.

\section{The interstellar spectrum}

\subsection{Interstellar absorption lines}

The rest-frame UV spectrum of the 8 o' clock LBG is dominated by interstellar absorption lines. Table 3 lists all the ISM lines detected and measured in our X-shooter spectra. Together with Ly $\alpha$ (which will be discussed separately in Sect. 5), we cover 31 transitions of elements from $\mathrm{H}$ to $\mathrm{Zn}$ in a variety of ionization stages from neutral (H I, O I) to highly ionized species ( C IV, N V). The low-ionization features are associated with neutral gas, while the high-ionization features predominantly trace gas at $T \geq 10^{4} \mathrm{~K}$ which is ionized by a combination of radiation from massive stars and collisional processes. All the interstellar lines are seen against the continuum provided by the integrated light of $\mathrm{O}$ and $\mathrm{B}$ stars in the galaxy. Vacuum rest-frame wavelengths, $\lambda_{\mathrm{lab}}$, and oscillator strengths, $f$, of the transitions are from the compilation by Morton (2003) and Jenkins \& Tripp (2006). The rest-frame equivalent widths, $W_{0}$, with their $1 \sigma$ errors were measured by summing the absorption over fixed velocity ranges, $\Delta v$. These velocity ranges were chosen to encompass the full extent of the absorption while minimizing the amount of continuum included.

Figures 5 and 6 show, respectively, the velocity profiles of most of the low- and high-ionization ISM absorption lines detected. We can note that the ISM lines in the 8 o'clock arc are very broad with absorption in the strongest transitions extending over a velocity range $\Delta v \sim 1300 \mathrm{~km} \mathrm{~s}^{-1}$, from about -985 to $+315 \mathrm{~km} \mathrm{~s}^{-1}$ relative to $z_{\text {sys }}=2.7350$. All the ion stages observed show similar absorption profiles: (i) they span the same overall velocity range (except the C IV doublet which is much broader, but it is also much stronger than the strongest lowionization lines); and (ii) they are characterized by the same two main absorption components with the major optical depth extending from -450 to $+50 \mathrm{~km} \mathrm{~s}^{-1}$ and the minor one being located at about $+120 \mathrm{~km} \mathrm{~s}^{-1}$.

Gas at the peak optical depth in the most clearly defined line profiles of the 8 o' clock arc (the unsaturated Si II $\lambda 1808$, Fe II $\lambda 1608$, and Fe II $\lambda 2374$ lines) occurs at $z_{\text {ISM }}=2.7335 \pm$ 0.0005 . This leads to a blueshift of the interstellar lines of $v_{\text {ISM }} \simeq$ $-120 \mathrm{~km} \mathrm{~s}^{-1}$ with respect to the stars and $z_{\text {sys. }}$. When redshifts of the centroid of the ISM lines ( $z_{\mathrm{abs}}$ values listed in Table 3 ) are considered, they give a mean $\left\langle z_{\text {abs }}\right\rangle=2.7324 \pm 0.0006$ and a velocity offset $v_{\text {ISM }} \simeq-209 \mathrm{~km} \mathrm{~s}^{-1}$. Finkelstein et al. (2009) derived a similar velocity offset $\left(-160 \mathrm{~km} \mathrm{~s}^{-1}\right)$ relative to the stars for the 8 o'clock arc. Such a blueshift of the interstellar gas is a common feature of star-forming galaxies at low as well as high redshifts (Heckman et al. 2000; Pettini et al. 2001; Shapley et al. 2003; Quider et al. 2009a). It is generally accepted that it results from large-scale outflows of the interstellar medium driven by the kinetic energy deposited by supernovae and the winds of massive stars. The outflow speed of $-120 \mathrm{~km} \mathrm{~s}^{-1}$ is typical of $z \sim 3$ LBGs for which Shapley et al. (2003) derived a mean value of $-150 \pm 60 \mathrm{~km} \mathrm{~s}^{-1}$. The location and nature of the interstellar gas moving at positive velocities relative to the stars remain, on the other hand, unexplained.

The ISM lines in the ESI spectra of two other well-studied lensed LBGs, the Cosmic Horseshoe and MS 1512-cB58 analyzed by Quider et al. (2009a) and Pettini et al. (2002), respectively, have very comparable characteristics (broadness, velocity range relative to $z_{\text {sys }}$, etc.) to the 8 o'clock arc lines. The ISM lines of the Cosmic Eye (Quider et al. 2009b) have, on the other hand, more complex velocity profiles with two well separated components. Quider et al. (2009a,b), moreover, pointed out one noticeable difference between the Cosmic Horseshoe plus the Cosmic Eye and cB58 in the optical depth of the ISM lines. Whereas in cB58 the strongest ISM lines have saturated cores of zero residual intensity (at the resolution of the ESI spectra which is comparable to the one of the X-shooter spectra), the same transitions in the Horseshoe and Eye seem to reach a minimum residual intensity $I_{\mathrm{obs}} / I_{\mathrm{c}} \sim 0.4$ and $\sim 0.3$, respectively, where $I_{\mathrm{obs}}$ and $I_{\mathrm{c}}$ denote the relative intensities in the line and in the continuum, respectively. Quider et al. attributed this effect to the fact that the interstellar gas does not completely cover the $\mathrm{O}$ and B stars producing the UV continuum against which the absorption is seen. The 8 o'clock arc, similarly to cB58, does not show evidence of partial coverage, the strongest ISM lines reach the zero residual intensity in their cores $\left(I_{\mathrm{obs}} / I_{\mathrm{c}} \ll 0.1\right.$; see Figs. 5 and 6).

\subsection{Ion column densities}

Values of column density, $N_{\text {aod }}$, for ions listed in Table 3 were derived using the apparent optical depth method of Savage \& Sembach (1991). This method is applicable in the case of X-shooter spectra despite their intermediate resolution of $42 \mathrm{~km} \mathrm{~s}^{-1} F W H M$ at $6000 \AA$, because the profiles of the ISM absorption lines in the 8 o'clock arc seem to be fully resolved.

The apparent column density of an ion in each velocity bin, $N_{\mathrm{a}}(v)$ in units of atoms cm $\mathrm{cm}^{-2}\left(\mathrm{~km} \mathrm{~s}^{-1}\right)^{-1}$, is related to the apparent optical depth in that bin, $\tau_{\mathrm{a}}(v)$, by the expression

$N_{\mathrm{a}}(v)=\frac{m_{\mathrm{e}} c}{\pi \mathrm{e}^{2}} \frac{\tau_{\mathrm{a}}(v)}{f \lambda_{\mathrm{lab}}}=3.768 \times 10^{14} \frac{\tau_{\mathrm{a}}(v)}{f \lambda_{\mathrm{lab}}(\AA)}$, 
M. Dessauges-Zavadsky et al.: Rest-frame UV spectrum of the 8 o'clock arc
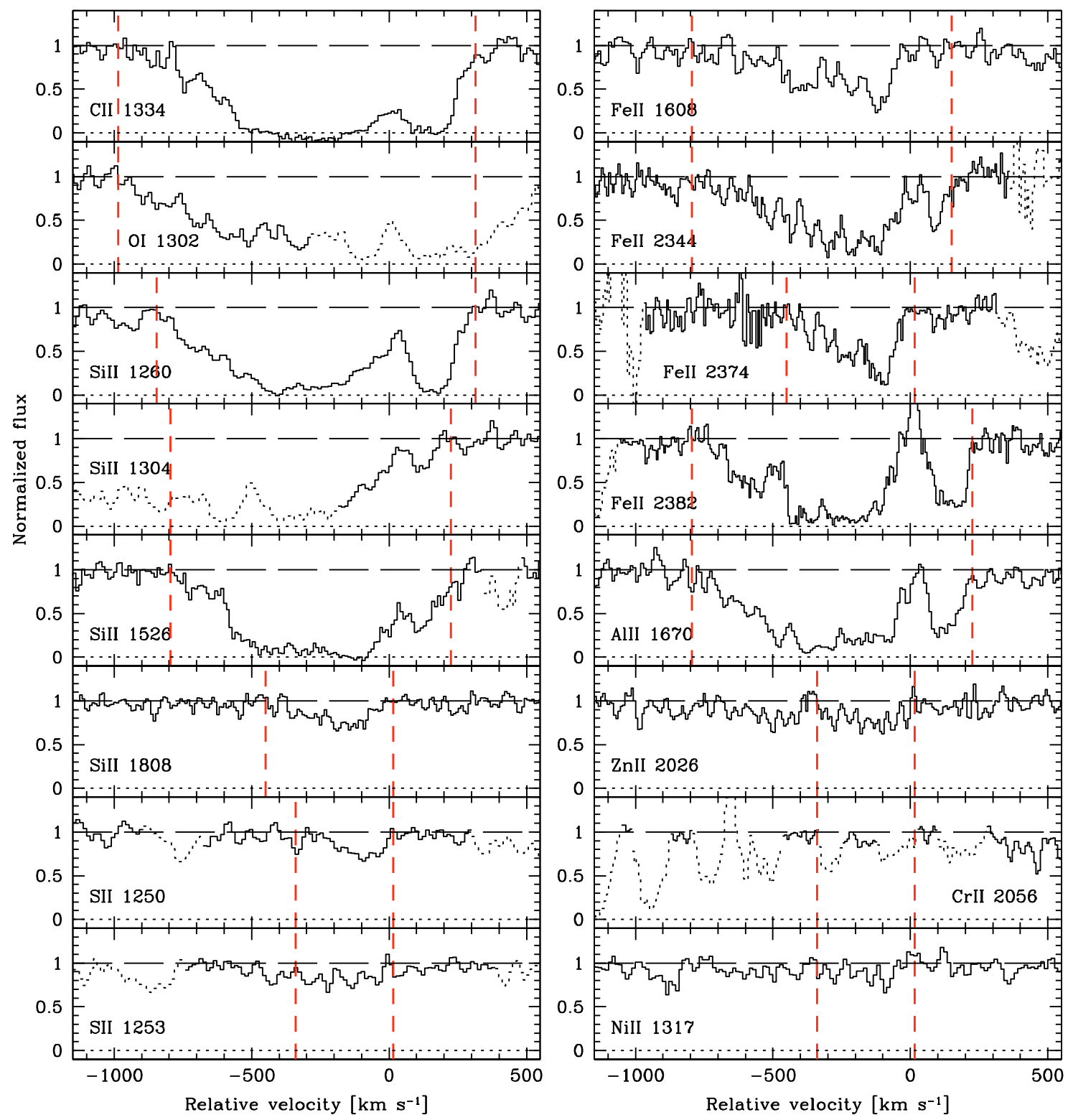

Fig. 5. Velocity plots of normalized profiles of the low-ionization interstellar absorption lines detected in the 8 o'clock arc. The zero velocity is fixed to $z_{\mathrm{sys}}=2.7350$. The vertical red dashed lines indicate the velocity range over which the equivalent widths listed in Table 3 are measured. The dotted lines are used to indicate blends with absorption features that are not those labeled in each panel.

where $f$ is the oscillator strength of the transition at the wavelength $\lambda_{\text {lab }}$ in $\AA$. The apparent optical depth is deduced directly from the observed intensity in the line at velocity $v, I_{\mathrm{obs}}(v)$, by

$\tau_{\mathrm{a}}(v)=-\ln \left[I_{\mathrm{obs}}(v) / I_{\mathrm{c}}(v)\right]$,

where $I_{\mathrm{c}}$ is the intensity in the continuum. The total column density of an ion $\mathrm{X}, N_{\mathrm{aod}}(\mathrm{X})$, is obtained by summation of Eq. (1) over the velocity interval where the line absorption takes place.

The apparent optical depth method provides, in addition, a stringent consistency check when several ISM lines arising from the same ground state of an ion but with different values of the product $f \lambda_{\text {lab }}$ are analyzed. The run of $N_{\mathrm{a}}(v)$ with $v$ should be the same for all such lines. In general, this condition will not be satisfied if there are saturated components in the absorption lines: the deduced value of $N_{\mathrm{a}}(v)$ will appear smaller for lines with higher values of $f \lambda_{\text {lab }}$. A similar effect can also appear if the covering of the integrated stellar continuum by the interstellar absorbing gas is inhomogeneous at a given velocity. The apparent optical depth method will yield discordant values of column density for different transitions of the same ion at that velocity.

In Fig. 7 we show the run of $N_{\mathrm{a}}(v)$ with $v$ for lines of interest. The four Si II lines detected have the largest dynamical range in $f \lambda_{\text {lab }}$ values of a factor of 320 from the weakest Si II $\lambda 1808$ to the strongest Si II $\lambda 1260$ transition. It can be seen from the plots that the absorption in the Si II lines at velocities between about -500 to $-100 \mathrm{~km} \mathrm{~s}^{-1}$ does not satisfy the consistency check discussed above, in that $N_{\mathrm{a}}(v)$ decreases with increasing $f \lambda_{\text {lab }}$. The effect is particularly dramatic in the core of the line profile centered at $v \simeq-120 \mathrm{~km} \mathrm{~s}^{-1}$. This could be indication of either saturated absorption components or inhomogeneous coverage of the stellar continuum. Saturation is undoubtably at play in Si II $\lambda 1260$, Si II $\lambda 1304$, and Si II $\lambda 1526$, because all the strongest ISM lines reach the zero residual intensity at these velocities (see Figs. 5 and 6). This is further supported by the Fe II lines. Indeed, with a range in $f \lambda_{\text {lab }}$ of a factor of 10 from Fe II $\lambda 2374$ to $\mathrm{Fe}$ II $\lambda 2382$, 

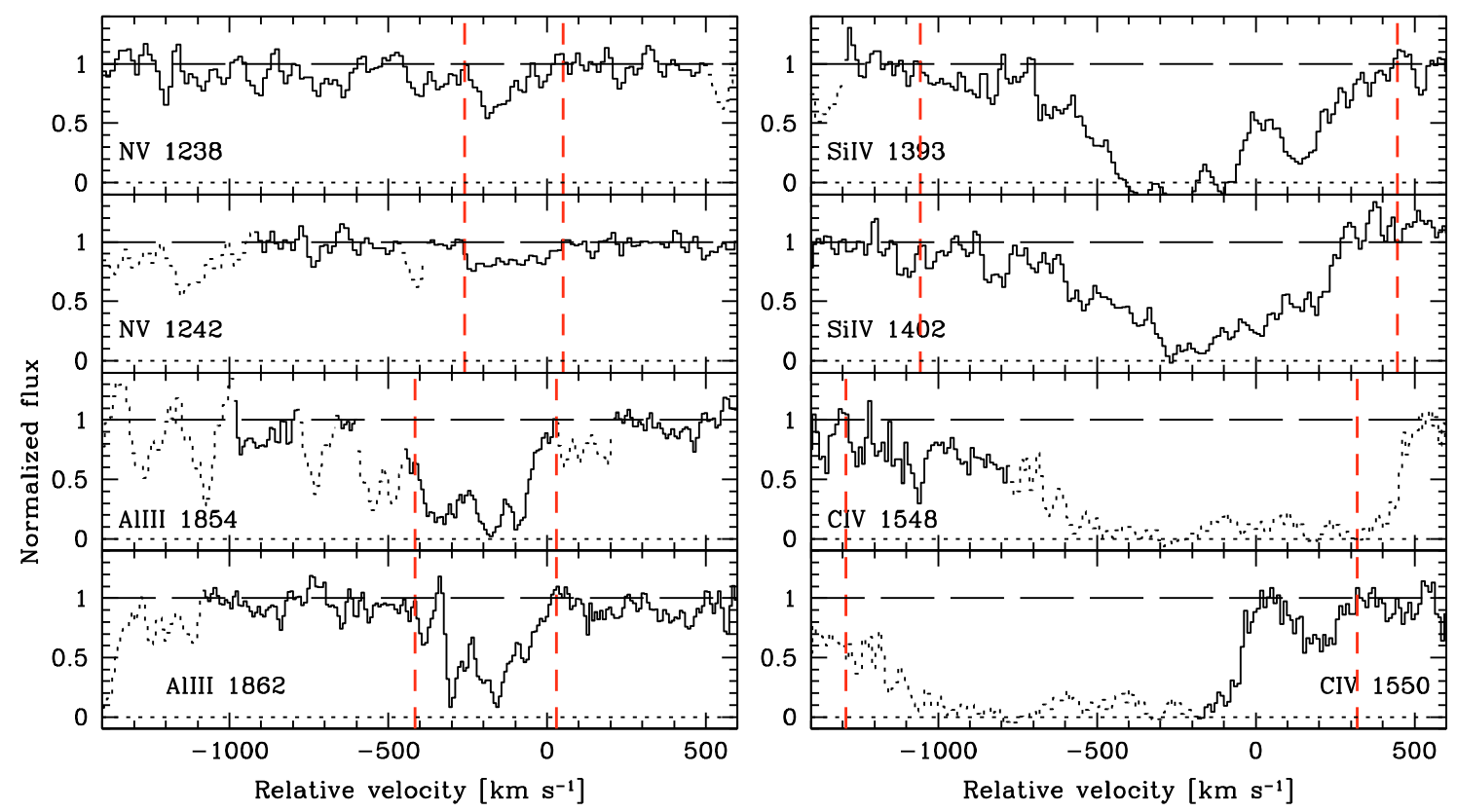

Fig. 6. Same as Fig. 5, but for the normalized profiles of the high-ionization interstellar absorption lines detected in the 8 o'clock arc.
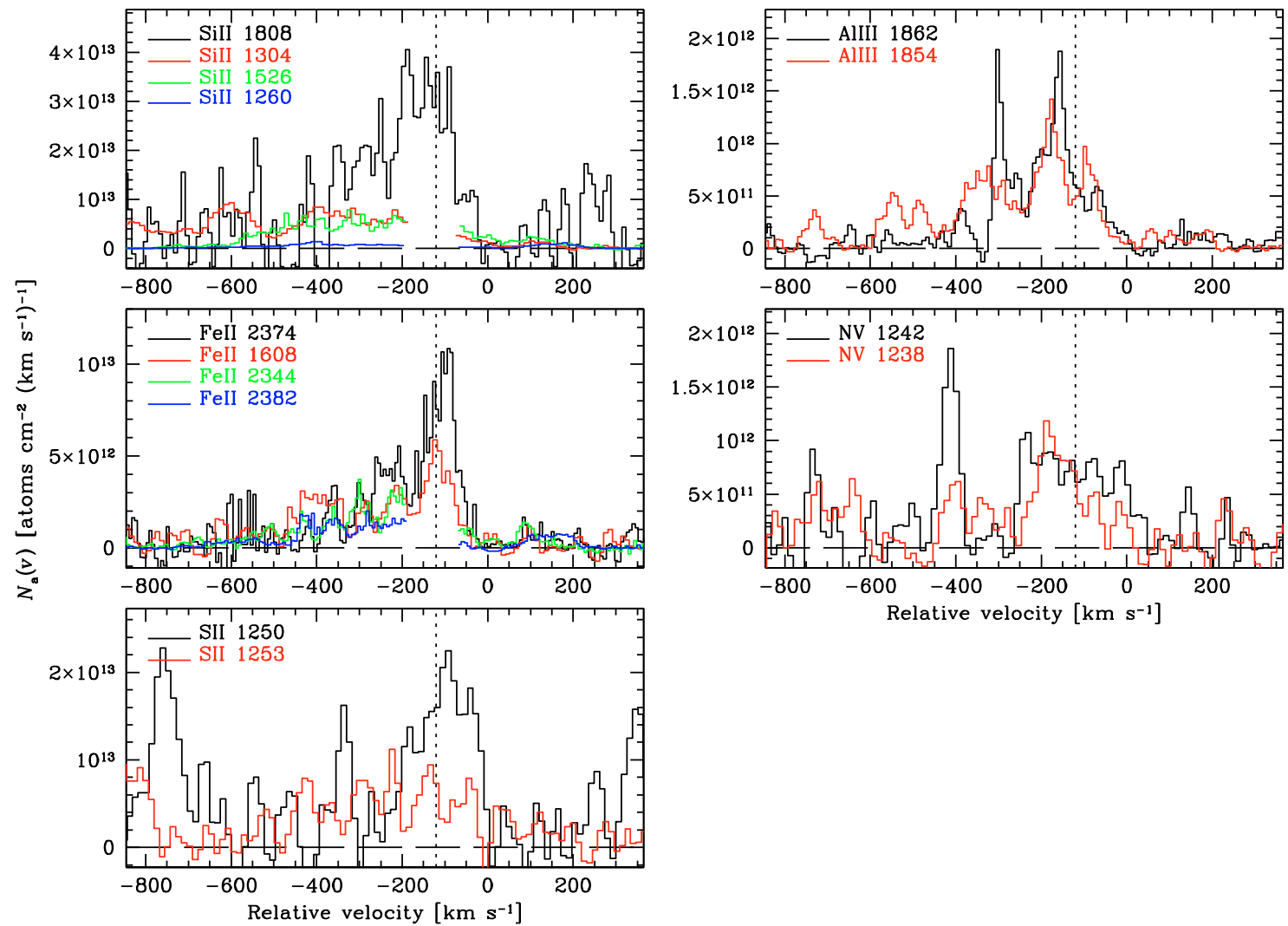

Fig. 7. Run of apparent column density, $N_{\mathrm{a}}(v)$, as a function of velocity relative to $z_{\text {sys }}=2.7350$ for absorption lines of various ions detected in the 8 o'clock arc. The transitions of a given ion are shown in increasing order of $f \lambda_{\text {lab }}$, starting from the top. The vertical dotted lines at $v_{\text {ISM }}=-120 \mathrm{~km} \mathrm{~s}^{-1}$ indicate the redshift of ISM gas at the peak optical depth, $z_{\text {ISM }}$.

all the Fe II lines satisfy relatively well the consistency check over the whole line profile, except in the line core, leaving not much place for partial coverage. The discrepancy in the line core between Fe II $\lambda 2374$ and Fe II $\lambda 1608$, two lines differing by only a factor of 1.28 in their $f \lambda_{\text {lab }}$ values, is already presumably indicative of saturation in Fe II $\lambda 1608$; and this similarly for the discrepancy between the two S II lines.
Finally, in the right panels of Fig. 7, we reproduce the run $N_{\mathrm{a}}(v)$ with $v$ for the lines of two high-ions, Al III and N v. For both ions we observe a very good consistency between their respective line transitions with $f \lambda_{\text {lab }}$ values differing by a factor of 2 . Hence, neither the saturation nor the partial coverage seem to affect the line profiles of the high-ions. 
M. Dessauges-Zavadsky et al.: Rest-frame UV spectrum of the 8 o' clock arc
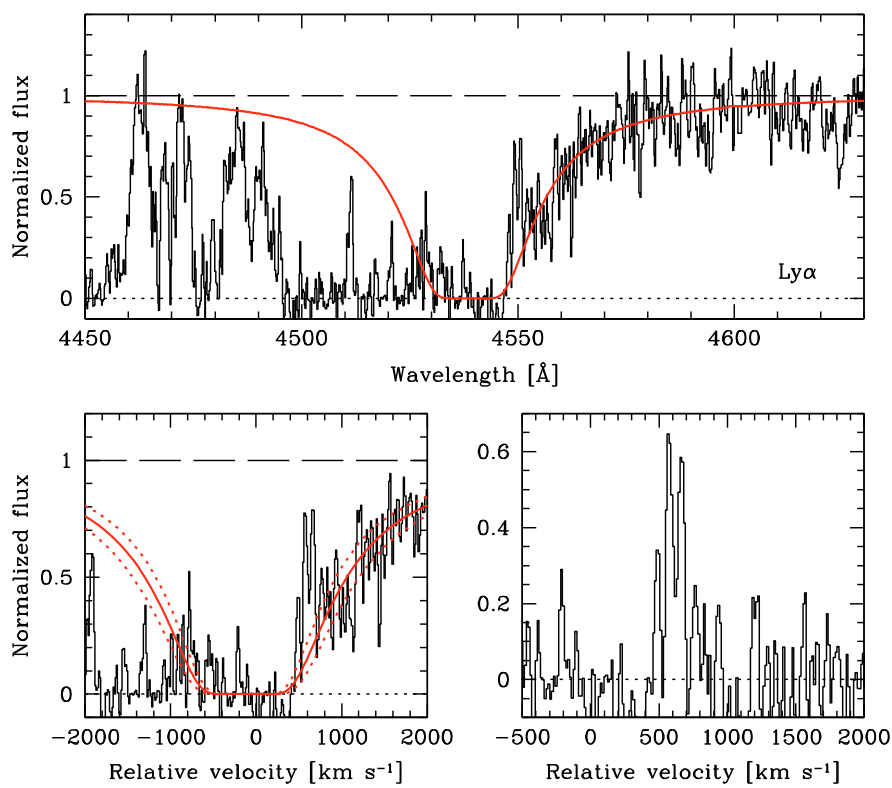

Fig. 8. Top panel: Normalized profile of the Ly $\alpha$ line in the 8 o'clock arc with the best Voigt profile fit (red solid line) obtained with $N(\mathrm{HI})=$ $3.7 \times 10^{20} \mathrm{~cm}^{-2}$. Bottom left-hand panel: Same as top panel, but showing a zoom of the $\operatorname{Ly} \alpha$ profile in velocity space with the zero velocity fixed to $z_{\text {sys }}=2.7350$. Also shown is the $1 \sigma$ error (red dotted line) on the fit of the damped Ly $\alpha$ profile with $N(\mathrm{HI})=2.9 \times 10^{20}$ and $4.5 \times$ $10^{20} \mathrm{~cm}^{-2}$. Bottom right-hand panel: Residual Ly $\alpha$ emission after subtraction of the $N(\mathrm{HI})=3.7 \times 10^{20} \mathrm{~cm}^{-2}$ damped absorption. The zero velocity is still fixed to $z_{\text {sys }}=2.7350$.

Table 3 lists the values of ion column density, $N_{\text {aod }}$, with the $1 \sigma$ error, determined by integrating Eq. (1) over the velocity interval, $\Delta v$, spanned by the respective absorption lines (same velocity intervals as those used for the equivalent width measurements). The lower limits refer to the saturated lines, and the values reported as upper limits mainly refer to non-detections. The saturation of a line is diagnosed via the consistency check discussed above. $N_{\text {adopt }}$ gives the adopted column density for a given ion. It corresponds either to the mean of $N_{\text {aod }}$ measurements obtained from unsaturated lines of a given ion, or to the most stringent limit in case of saturated lines and non-detections. We have, nevertheless, to keep in mind that even for ions for which we cover at least one transition that is sufficiently weak for the apparent optical depth method to be applicable, the derived column density values may be underestimated, because of possible saturation in the line core and possible inhomogeneous coverage of the integrated stellar UV light (and saturation) in the line wings.

\section{The Ly $\alpha$ line}

\subsection{The damped Ly $\alpha$ profile}

The Ly $\alpha$ line profile in the 8 o'clock LBG is a combination of absorption and emission. In Fig. 8 we show the Ly $\alpha$ line profile and our decomposition of this feature. The absorption component is best fitted by a damped profile. We used the software FITLYMAN in MIDAS (Fontana \& Ballester 1995) which generates theoretical Voigt profiles and performs $\chi^{2}$ minimizations to fit the Ly $\alpha$ profile. The damping wings (and in particular the red wing, the blue wing being less constraining and more noisy) are well fitted with a neutral hydrogen column density $N(\mathrm{H} \mathrm{I})=(3.7 \pm 0.8) \times 10^{20} \mathrm{~cm}^{-2}$ centered at $v_{\text {ISM }} \simeq-120 \mathrm{~km} \mathrm{~s}^{-1}$,
Table 4. Relative velocities in the 8 o'clock arc.

\begin{tabular}{lcc}
\hline \hline Spectral features & $z^{a}$ & $\begin{array}{c}v^{b} \\
\left(\mathrm{~km} \mathrm{~s}^{-1}\right)\end{array}$ \\
\hline Stellar photospheric absorption lines & $z_{\text {stars }}=2.7347$ & -24 \\
H II emission lines & $z_{\mathrm{C} \text { III }}=2.7350$ & 0 \\
Recombination emission line & $z_{\mathrm{Si} \text { II }}=2.7352$ & +16 \\
Interstellar absorption lines & $z_{\mathrm{ISM}}=2.7335$ & -120 \\
Ly $\alpha$ emission line & $z_{\mathrm{Ly} \alpha}=2.7420$ & +570 \\
\hline
\end{tabular}

Notes. ${ }^{(a)}$ Vacuum heliocentric; ${ }^{(b)}$ relative to the systemic redshift $z_{\text {sys }}=$ 2.7350 that is the mean of $z_{\mathrm{stars}}$ and $z_{\mathrm{em}}$ (the mean redshift of emission lines).

the velocity where the ISM lines have the largest optical depth. The derived H I column density is typical of values observed in damped Ly $\alpha$ absorption line systems (Prochaska et al. 2005; Noterdaeme et al. 2009). With the other three H I column densities, $N(\mathrm{HI})=(7.0 \pm 1.5) \times 10^{20},(2.5 \pm 1.0) \times 10^{21}$, and $(3.0 \pm 0.8) \times 10^{21} \mathrm{~cm}^{-2}$ measured in the lensed LBGs MS 1512cB58 (Pettini et al. 2002), FOR J0332-3557 (Cabanac et al. 2008), and the Cosmic Eye (Quider et al. 2009b), respectively, and the $\mathrm{H}$ I column density measurements for $11 \mathrm{LBGs}$ obtained by Verhamme et al. (2008) with the help of their 3D Ly $\alpha$ radiation transfer code, the Lyman Break galaxies exhibit a large range of $N(\mathrm{HI})$ values, with no trend to a particularly extreme reservoir of neutral gas.

Subtraction of the fit of the damped $\operatorname{Ly} \alpha$ absorption reveals a weak Ly $\alpha$ emission line (bottom right-hand panel in Fig. 8). The emission is the strongest near $v_{\mathrm{Ly} \alpha} \simeq+570 \mathrm{~km} \mathrm{~s}^{-1}$, i.e. redshifted relative to the systemic redshift of the galaxy. It exhibits an asymmetric profile with a relatively abrupt drop on the blue side and a more gradual decrease at $v \gtrsim+700 \mathrm{~km} \mathrm{~s}^{-1}$ extending up to $v \sim+1100 \mathrm{~km} \mathrm{~s}^{-1}$. The rest-frame equivalent width of this Ly $\alpha$ emission line, integrated over the velocity interval $v=+400$ to $+1100 \mathrm{~km} \mathrm{~s}^{-1}$, is $W_{0}(\mathrm{Ly} \alpha)=-0.35 \AA$. However, as radiation transfer models show (see Sect. 5.2), this emission peak traces only a very small fraction of the intrinsic Ly $\alpha$ emission of the source. Table 4 summarizes the relative velocity measurements obtained for the various spectral features in the 8 o' clock arc.

The Ly $\alpha$ emission profile in the 8 o'clock arc is remarkably similar to that of MS 1512-cB58 (Pettini et al. 2000, 2002), except that the emission in the 8 o'clock arc is more redshifted than in cB58 by $>200 \mathrm{~km} \mathrm{~s}^{-1}$. The lensed LBG studied by Cabanac et al. (2008) exhibits an even more redshifted emission peaking at about $+720 \mathrm{~km} \mathrm{~s}^{-1}$. Redshifted Ly $\alpha$ emission is often seen in high-redshift galaxies and in local H II and starburst galaxies. This redshift results from large-scale outflows of the interstellar media. Indeed, Ly $\alpha$ emission is suppressed by resonant scattering and the only Ly $\alpha$ photons that can escape unabsorbed in the observer's direction are those backscattered from the far side of the expanding nebula, whereas in absorption against the stellar continuum, we see the approaching part of the outflow. The velocity offset between the Ly $\alpha$ emission and low-ionization ISM lines measured in the 8 o' clock arc is typical of $z \sim 3$ LBGs in general, and agrees well with the offset observed for LBGs with the strongest Ly $\alpha$ absorption (Shapley et al. 2003).

\subsection{Radiation transfer modeling}

\subsubsection{MCLya code and input parameters}

To model the Ly $\alpha$ line of the 8 o'clock arc, we use an improved version of the Monte Carlo radiation transfer code, MCLya, 
of Verhamme et al. (2006) including the detailed physics of Ly $\alpha$ line and UV continuum transfer, dust scattering, and dust absorption for arbitrary 3D geometries and velocity fields. The following improvements have been included: angular redistribution functions taking quantum mechanical results for Ly $\alpha$ into account (Stenflo 1980; Dijkstra \& Loeb 2008), frequency changes of Ly $\alpha$ photons due to the recoil effect (e.g., Zheng \& Miralda-Escudé 2002), the presence of deuterium (assuming a canonical abundance of $\mathrm{D} / \mathrm{H}=3 \times 10^{-5}$, Dijkstra et al. 2006), and anisotropic dust scattering using the Henyey-Greenstein phase function (with parameters as adopted in Witt \& Gordon (2000)). Furthermore, a relatively minor bug in the angular redistribution of $\operatorname{Ly} \alpha$ hotons has been fixed, and the code has been parallelized for efficient use on supercomputers. For the physical conditions used in the simulations of the present paper, these improvements lead only to minor changes with respect to the MCLya version used by Schaerer \& Verhamme (2008) and Verhamme et al. (2008). More details on the code upgrades will be given in Hayes et al. (2009, in preparation).

For simplicity and as in earlier modeling of $z \sim 3$ LBGs (Verhamme et al. 2008), we assume a simple geometry, i.e. a spherical, homogeneous shell of cold ISM (neutral hydrogen plus dust) surrounding the starburst (UV continuum plus Ly $\alpha$ line emission from the $\mathrm{H}$ II region). The input parameters of the 3D transfer simulations are: the radial expansion velocity, $v_{\text {exp }}$, the H I column density, $N(\mathrm{HI})$, the H I velocity dispersion, $b$, and the dust absorption optical depth, $\tau_{a}$, which expresses the dust-to-gas ratio. As discussed in Verhamme et al. (2006), $\tau_{a}$ is related to the usual color excess $E(B-V)$ by $E(B-V) \approx(0.06 \ldots 0.11) \tau_{a}$. We assume $E(B-V)=0.1 \tau_{a}$ for convenience.

For each parameter set, a full Monte Carlo (MC) simulation is run. As described in Verhamme et al. (2006), our MC simulations are computed for a flat input spectrum, keeping track of the necessary information to recompute a posteriori simulations for arbitrary input spectra. For the Ly $\alpha$ fits, we assume an input spectrum given by a flat (stellar) continuum plus the Ly $\alpha$ line described by a Gaussian with variable equivalent width, $W(\operatorname{Ly} \alpha)$, and full width at half maximum, $F W H M(\operatorname{Ly} \alpha)$. In a first step, we use an automatic line profile fitting tool, relying on an extensive grid of $5200 \mathrm{MC}$ simulations and exploring the full parameter space (Hayes et al. 2009, in preparation). Some of the model parameters (e.g., $v_{\text {exp }}$ ) are fixed in a subsequent step to take observational constraints into account. Shortward of $4528 \AA$ the blue wing of the Ly $\alpha$ line is affected by other lines (see Fig. 8) and is therefore excluded from our line fitting procedure.

\subsubsection{Results}

Our automated Ly $\alpha$ fitting code yields the following best-fit parameters for the Ly $\alpha$ line profile of the 8 o'clock arc: $v_{\exp }=$ $0_{-0}^{+52} \mathrm{~km} \mathrm{~s}^{-1}, \log N(\mathrm{HI})=20.8_{-0.03}^{+0.6} \mathrm{~cm}^{-2}, \tau_{a}=3_{-2.8}^{+0.96}, b=$ $40_{-30}^{+46} \mathrm{~km} \mathrm{~s}^{-1}, W(\mathrm{Ly} \alpha)=-300_{-0}^{+299} \AA$, and $F W H M(\operatorname{Ly} \alpha)=$ $100_{-49}^{+0} \mathrm{~km} \mathrm{~s}^{-1}$, where the errors correspond to the formal 1dimensional $68 \%$ confidence levels. Here, negative equivalent widths indicate emission. Although these formal errors are quite large, one should notice that strong correlations exist between various parameters. For example, reducing the dust content $\left(\tau_{a}\right)$ requires a higher $\mathrm{H}$ I column density to maintain the same width of the broad Ly $\alpha$ absorption.

The most interesting result is the finding of a high $\mathrm{HI}$ column density and a large dust optical depth, which (for the closed geometry adopted here) are necessary to create a damped

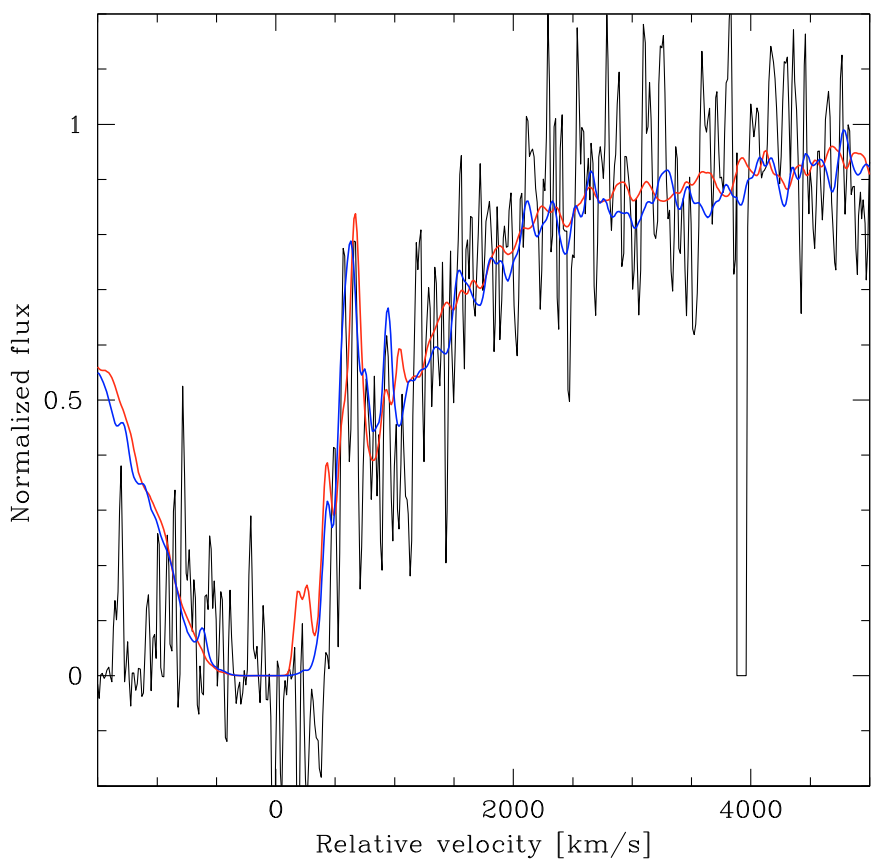

Fig. 9. Ly $\alpha$ line profile fits (red/blue) to the observed profile (black) of the 8 o'clock arc using a modified version of the 3D Ly $\alpha$ and dust radiation transfer code of Verhamme et al. (2006) applied to homogeneous, spherically expanding shells surrounding a UV continuum + line source. The redshift and the radial outflow velocity are fixed at $z=z_{\text {sys }}$ and $v_{\text {exp }}=150 \mathrm{~km} \mathrm{~s}^{-1}$, respectively, as determined from the observations. The remaining fit parameters are $\log N(\mathrm{HI})=20.8(20.8) \mathrm{cm}^{-2}$, $\tau_{a}=3(4), b=20(80) \mathrm{km} \mathrm{s}^{-1}, W(\operatorname{Ly} \alpha)=-50(-50) \AA$, and $F W H M(\operatorname{Ly} \alpha)=50(160) \mathrm{km} \mathrm{s}^{-1}$ for the red (blue) curves, respectively.

Ly $\alpha$ absorption line, as already shown in Verhamme et al. (2006) and Schaerer \& Verhamme (2008). The derived H I column density exceeds the one determined in Sect. 5.1 from a simple Voigt profile fitting by a factor of $\sim 1.7$, again this is expected given the assumption on the geometry of a shell surrounding the UV source (Verhamme et al. 2006). This difference in the N(H I) estimates (although geometry dependent) has implications on the abundance determinations discussed in Sect. 6.1. The best-fit value of $\tau_{a}$ corresponds to an $\mathrm{UV}$ attenuation $E(B-V) \approx 0.3$, in reasonable agreement with the color excess $E(B-V)=0.67 \pm$ 0.21 derived from the Balmer decrement by Finkelstein et al. (2009).

Despite an overall good fit of the damped Ly $\alpha$ absorption component in the 8 o'clock arc, the above solution fails to reproduce the $\operatorname{Ly} \alpha$ emission component observed in the red wing of the Ly $\alpha$ absorption (see Sect. 5.1 and Fig. 8). However, this emission is easily recovered when assuming larger expansion velocities. In particular, for $v_{\exp } \sim 100-150 \mathrm{~km} \mathrm{~s}^{-1}$, an emission peak appears naturally in the red wing and at the observed wavelength. Two examples of such fits are shown in Fig. 9. This bulk radial velocity of the shell is in agreement with the mean outflow velocity of $-120 \mathrm{~km} \mathrm{~s}^{-1}$ measured from the lowionization interstellar absorption lines relative to the systemic redshift (see Sect. 4.1 and Table 4). For higher velocities, the peak is shifted too far to the red. The input, i.e. intrinsic FWHM of the Ly $\alpha$ emission line before undergoing radiation transfer, does not much affect the result. Adopting e.g. FWHM $(\operatorname{Ly} \alpha)=$ $160 \mathrm{~km} \mathrm{~s}^{-1}$, as suggested by the C III] lines, does not alter the resulting profile. The results are more sensitive to $b$, which describes the adopted velocity dispersion in the expanding shell. Good fits are obtained with $b \lesssim 90 \mathrm{~km} \mathrm{~s}^{-1}$, as also shown in 
Fig. 9. Larger values of $b$ lead to a broader Ly $\alpha$ emission peak, when strong intrinsic $\operatorname{Ly} \alpha$ emission is present. These values for $b$, corresponding to $F W H M \lesssim 210 \mathrm{~km} \mathrm{~s}^{-1}$, are reasonable, as can be judged from the column density weighted velocity distribution of the low-ionization ISM lines (see Fig. 7). From this we conclude that our radiation transfer models, assuming a simple expanding shell and ISM properties in good agreement with observations, are able to reproduce the observed Ly $\alpha$ profile, including the broad absorption and the small peak of "reminiscent" Ly $\alpha$ emission. More sophisticated models would require a knowledge of the presumably more complex ISM geometry and velocity field.

The Ly $\alpha$ profile fits of the 8 o'clock arc shown in Fig. 9 correspond to an input intrinsic $\operatorname{Ly} \alpha$ emission with a rest-frame equivalent width of $W_{0}(\operatorname{Ly} \alpha)=-50 \AA$, a typical value expected when star formation extends over timescales longer than $\gtrsim 10^{7} \mathrm{yr}$. The Ly $\alpha$ fit for this LBG therefore agrees well with our earlier findings for other LBGs (Schaerer \& Verhamme 2008; Verhamme et al. 2008). In particular, our radiation transfer models show that the Ly $\alpha$ line profile of the 8 o'clock arc is compatible with an approximately constant star formation, where the intrinsic Ly $\alpha$ emission is transformed into the complex, observed profile by radiation transfer effects and absorption by dust.

The Ly $\alpha$ emission peak in the red wing of the $\operatorname{Ly} \alpha$ absorption is the result of multiply backscattered Ly $\alpha$ line photons emitted in the $\mathrm{H}$ II region surrounded by the cold, expanding shell, as explained in Schaerer \& Verhamme (2008) for cB58. The velocity comparison of the observed Ly $\alpha$ emission peak $\left(500-700 \mathrm{~km} \mathrm{~s}^{-1}\right)$ to the outflow velocity $\left(120 \mathrm{~km} \mathrm{~s}^{-1}\right)$ in the 8 o'clock arc (see Table 4) indicates that these emergent Ly $\alpha$ photons have benefited from multiple (2-3) backscattering across the shell. This relatively large number of backscattering is due to the high H I column density. It explains why the velocity shift of the Ly $\alpha$ emission peak is found at $v_{\text {Ly } \alpha} / v_{\exp } \sim 4-6$, larger than the "typical" shift of $v_{\text {Ly } \alpha} / v_{\text {exp }} \sim 2$ suggested by Verhamme et al. (2006) for LBGs with lower $N(\mathrm{H} \mathrm{I})$.

The observed velocity offset between the Ly $\alpha$ emission peak and the low-ionization ISM lines of $\Delta v($ Ly $\alpha-$ ISM $) \sim$ $620-820 \mathrm{~km} \mathrm{~s}^{-1}$ in the 8 o'clock arc is also in good agreement with the average $\Delta v(\operatorname{Ly} \alpha-$ ISM $) \sim 790 \mathrm{~km} \mathrm{~s}^{-1}$ measured from the composite spectra of $z \sim 3$ LBGs with strong Ly $\alpha$ absorption (see Shapley et al. 2003). For cB58, in contrast, $\Delta v(\operatorname{Ly} \alpha-$ ISM $) \sim 550 \mathrm{~km} \mathrm{~s}^{-1}$. In this respect, the 8 o'clock arc appears to be more typical of the category of LBGs with damped Ly $\alpha$ profiles, and our modeling results for this LBG therefore support the explanation of Verhamme et al. (2008) for the observed correlation between $\Delta v(\operatorname{Ly} \alpha-\mathrm{ISM})$ and $W_{0}(\mathrm{Ly} \alpha)$ shown by Shapley et al. (2003). In short, variations of the velocity offset between the Ly $\alpha$ emission and the ISM absorption lines are strongly affected by the interstellar medium column density and do not primarily reflect changes in outflow velocities.

\section{Summary of the results and discussion}

\subsection{Consistency in the various metallicity estimates}

Lensed Lyman Break galaxies observed with current instrumentation are the only objects at high redshifts where metallicity estimates from stars, H II regions, and interstellar gas are all accessible. They thus offer a nice comparison of these various environments. In Table 5 we summarize all the available metallicity estimates in the 8 o'clock arc.

We determine the metallicity of OB stars from the photospheric absorption lines and the related "1370", "1425", and "1978" metallicity indices (Sect. 3.2). The well-calibrated
Table 5. Metallicity estimates in the 8 o'clock arc.

\begin{tabular}{lclc}
\hline \hline Environment & Method & Elements & $Z / Z_{\odot}$ \\
\hline H II regions $^{a}$ & $\mathrm{~N} 2$ & $\mathrm{O}$ & 0.83 \\
H II regions & O3N2 & $\mathrm{O}$ & 0.39 \\
OB stars $^{b}$ & "1425" index & $\mathrm{C}, \mathrm{Si}, \mathrm{Fe}$ & 0.85 \\
OB stars $^{b}$ & "1978" index & $\mathrm{Fe}$ & 0.79 \\
ISM gas $^{c}$ & Apparent optical depth & $\mathrm{Si}$ & 0.65 \\
\hline
\end{tabular}

Notes. (a) As derived by Finkelstein et al. (2009); ${ }^{(b)}$ this work (Sect. 3.2); ${ }^{(c)}$ this work (Sect. 4.2).

"1425" and " 1978 " indices lead to metallicities, $Z=0.85 Z_{\odot}$ and $Z=0.79 Z_{\odot}$, respectively, which are in very good mutual agreement. Finkelstein et al. (2009) obtained the oxygen abundance of the ionized gas from nebular emission lines redshifted into the NIR, using the N2 and O3N2 indices of Pettini $\&$ Pagel (2004). The derived O3N2 metallicity is only half of the $\mathrm{N} 2$ metallicity. The authors assigned this discrepancy to the uncertainty in their [O III] flux measurement, and consider the N2 metallicity, $Z=0.83 Z_{\odot}$, as being the more reliable. The metallicity of early-type stars thus is in excellent agreement with that of the HII regions that surround the stars. We naturally expect the two metallicities to be the same, since the stars presumably formed very recently out of the gas which they now ionize. The corresponding mean metallicity is $Z=0.82 Z_{\odot}$.

The 8 o'clock arc with this metallicity and its stellar mass estimate of $\sim 4.2 \times 10^{11} M_{\odot}$ (Finkelstein et al. 2009) is consistent with the mass-metallicity relation at $z \sim 2.2$ of Erb et al. (2006b) ${ }^{4}$, although it lies at a slightly higher mass than the highest mass points of Erb et al. We may have expected the 8 o'clock arc to fall below the $z \sim 2.2$ trend, given its higher redshift and the observed trend in the mass-metallicity relation to move downward in metallicity objects from low-to-high redshifts (Maiolino et al. 2008). However, this is not the case, as also stated by Finkelstein et al. (2009).

We also determine the metallicity in the interstellar medium of the 8 o'clock arc from the numerous ISM absorption lines detected (Sect. 4.2). The $Z=0.65 Z_{\odot}$ metallicity of the ISM gas, as determined from the silicon abundance, is about $80 \%$ the metallicity of OB stars and ionized gas, i.e. only $\sim 0.1$ dex lower. When taking these measurements at face value, they suggest that the interstellar medium of the 8 o'clock LBG has rapidly been polluted by ejecta from $\mathrm{OB}$ stars and enriched to the metallicity of H II regions. On the other hand, several uncertainties affect these measurements: (i) the radiation transfer modeling seems to show that the H I column density is underestimated by a factor of $\sim 1.7$ when derived from a simple Voigt profile fitting of the damped Ly $\alpha$ profile (see Sect. 5.2), if this is correct the ISM gas metallicity would also be a factor of $\sim 1.7$ lower than the stellar and ionized gas metallicities; (ii) without the possibility to carry out a photoionization analysis, we assume that $\mathrm{Si}$ II/H I $=\mathrm{Si} / \mathrm{H}$, but there may be some need for ionization corrections; and (iii) some saturation in the line cores and a possible inhomogeneous coverage of the stellar light in the line wings may lead to an underestimation of the metal column densities. How significant these effects are, remains difficult to quantify with our data. Metallicity differences are observed between the ionized gas and the neutral ISM gas in some nearby dwarf galaxies (see e.g., Aloisi et al. 2003; Lebouteiller et al. 2009), but even these local examples are of controversial interpretation.

\footnotetext{
${ }^{4}$ Established on the same metallicity calibration index N2 as the one used by Finkelstein et al. (2009) for their metallicity estimate.
} 


\subsection{Elemental abundances in the interstellar medium}

From the ion column densities (Sect. 4.2) and the H I column density derived from the damped $\operatorname{Ly} \alpha$ profile (Sect. 5.1), we determine the chemical abundances of several elements in the interstellar medium of the 8 o'clock arc. They are listed, with their $1 \sigma$ errors, in the last two columns in Table 3 relative to the solar meteoritic abundance scale from Grevesse et al. (2007).

Our X-shooter observations cover three $\alpha$-capture elements, $\mathrm{Si}, \mathrm{S}$, and $\mathrm{O}$. Si and $\mathrm{S}$ give a consistent picture within measurement uncertainties, with abundances $[\mathrm{Si} / \mathrm{H}]=-0.19 \pm 0.14$ and $[\mathrm{S} / \mathrm{H}]=-0.28 \pm 0.14$, respectively. The lower limit on the abundance of $\mathrm{O}$ is, on the other hand, useless, given the strong saturation of the O I $\lambda 1302$ line and its blend with Si II $\lambda 1304$.

As for the iron-peak elements, we have the abundance measurement of $\mathrm{Fe}$ and the upper limits on the abundances of $\mathrm{Zn}, \mathrm{Cr}$, and $\mathrm{Ni}$. The $\mathrm{Fe}$ abundance, $[\mathrm{Fe} / \mathrm{H}]=-0.88 \pm 0.15$, is lower than that of $\alpha$-elements by $0.69 \mathrm{dex}(\mathrm{Si})$. This underabundance could be a departure from the solar scale due to nucleosynthetic effects, a reflection of depletion of Fe onto dust grains, or both. The abundance of $\mathrm{Zn}$, an element that is undepleted, usually helps to break the above ambiguity (e.g., Pettini et al. 1999). Unfortunately, our super-solar $\mathrm{Zn}$ abundance, $[\mathrm{Zn} / \mathrm{H}]<+0.31$ (super-solar abundances are not observed for any other elements), favor that the detection of the Zn II $\lambda 2026$ line is extremely marginal (see Fig. 5) and that the derived $\mathrm{Zn}$ abundance is only a non-constraining upper limit.

The gas-phase abundance of the interstellar Fe does not also agree with the $\mathrm{Fe}$ abundance in the $\mathrm{OB}$ stars as deduced from the "1978" index which arises from the blending of numerous Fe III transitions (see Table 5) and which does not suffer from dust depletion. At face value, $(\mathrm{Fe} / \mathrm{H})_{\text {stars }} \simeq 6 \times(\mathrm{Fe} / \mathrm{H})_{\mathrm{ISM}}$. This could be an indication that most of the underabundance of the interstellar Fe in the 8 o'clock arc is due to dust depletion. If dust depletion is the sole origin of the $\mathrm{Fe}$ underabundance relative to the $\alpha$-elements, then, according to what is observed in the interstellar medium of the Milky Way (Savage \& Sembach 1996), $\mathrm{Si}$ should also be depleted. This is, however, not what is observed, as the abundance of $\mathrm{Si}$ is approximately the same to the abundance of the undepleted $\mathrm{S}$, as well as to the abundance of $\mathrm{O}$ as derived from $\mathrm{H}$ II regions and to the abundance in the OB stars (see Table 5). Nevertheless, the conditions in the ISM of these actively star-forming galaxies, that are the LBGs, are likely to be quite different from those in the Milky Way, where the star formation rate is about 100 times lower. We are hence unable to quantify the respective contributions from dust depletion and nucleosynthesis to the underabundance of Fe relative to the $\alpha$-elements. An intrinsic overabundance of the $\alpha$-capture products of type II supernovae relative to the iron-peak elements whose release into the ISM is delayed, because produced on much longer timescales by type Ia supernovae, would suggest a relatively young $(\$ 0.3-1 \mathrm{Gyr})$ age for the bulk of stars in the 8 o'clock LBG. Such an age limit is in agreement with the spectral energy distribution of the 8 o'clock arc (see Finkelstein et al. 2009) and with the strength of the He II $\lambda 1640$ emission line (see Sect. 3.3).

\subsection{Comparison with other Lyman Break galaxies}

The lensed 8 o'clock arc offers a new opportunity to compare the detailed properties of individual Lyman Break galaxies and establish how typical are the properties of MS 1512-cB58, the first lensed LBG studied (Pettini et al. 2000, 2002; Teplitz et al. 2000). Quider et al. (2009a,b) provided the analysis of the second and the third lensed LBGs, the Cosmic Horseshoe and the Cosmic Eye, respectively, made at a comparable precision thanks to their intermediate-resolution ESI spectra. Quider et al. (2009a) discuss the similarities and differences between the Horseshoe and cB58 in light of $z \sim 2-3$ star-forming galaxies. Here we would like to add the new example of the 8 o'clock arc.

\section{General characteristics}

After correction for lensing, with $\sim 11 L^{*}$ the 8 o'clock arc is the most luminous Lyman break galaxy relative to $\mathrm{cB58}$, the Horseshoe, and the Eye in the rest-frame UV (Allam et al. 2007). But, the lensed 8 o'clock LBG is not only among the most luminous LBGs. Dynamical masses of $M_{\text {dyn }} \sim 1-1.7 \times 10^{10} M_{\odot}$, typical of LBGs, are measured for cB58, the Horseshoe, and the Eye (see Teplitz et al. 2000; Coppin et al. 2007; Hainline et al. 2009). In comparison, the stellar mass of the 8 o'clock arc is estimated to $M_{\text {stars }} \sim 4.2 \times 10^{11} M_{\odot}$ (Finkelstein et al. 2009), namely significantly more massive. cB58 has the lower extinction $E(B-V) \sim 0.27$ compared to the Horseshoe and the 8 o'clock arc with $E(B-V) \sim 0.45$ and $\sim 0.67$, respectively, as derived from the Balmer decrement. The extinction corrected $\mathrm{H} \alpha$ and $\mathrm{H} \beta$ star formation rates of $\mathrm{cB} 58$, the Horseshoe, and the Eye are $S F R \sim 50-100 M_{\odot} \mathrm{yr}^{-1}$ (Stark et al. 2008; Quider et al. 2009a). The star formation rate of the 8 o'clock arc, $S F R \sim 270 M_{\odot} \mathrm{yr}^{-1}$, is significantly higher, namely higher than $\sim 85 \%$ of star-forming galaxies at $z \sim 2-3$ (Finkelstein et al. 2009).

\section{The metallicity}

The metallicities $\left(Z \simeq 0.4-0.5 Z_{\odot}\right)$ of cB58, the Horseshoe, and the Eye are very comparable, whereas the metallicity $(Z \sim$ $0.8 Z_{\odot}$ ) of the 8 o'clock arc is sensibly higher. This is inline with the mass-metallicity relation derived from samples of $z \sim 2.2$ and $z \sim 3.5$ star-forming galaxies (Erb et al. 2006b; Maiolino et al. 2008). Indeed, given the $\sim 11 L^{*}$ luminosity of the 8 o'clock arc and its high stellar mass, we expect it to have a metallicity higher than that for typical $L^{*}$ galaxies at this redshift. The 8 o'clock LBG, with its high metallicity and high mass, is located at the upper end of the LBG mass-metallicity distribution, and may hence appear as less representative of the whole LBG population. All the Lyman Break galaxies at $1<z<3$, with their metallicities in the range of $\sim 0.3$ to $0.9 Z_{\odot}$ seem to have already achieved a near-solar metallicity at relatively early times, some 12 Gyr ago in the case of the 8 o'clock arc, when the age of the Universe was only $17 \%$ of what it is today. This advanced degree of chemical enrichment is consistent with the original suggestion by Steidel et al. (1996) that LBGs are the progenitors of today's ellipticals and bulges, since such relatively high abundances are common in the most massive galaxies at $z \simeq 3$.

\section{The interstellar medium}

cB58, the Horseshoe, and the 8 o'clock arc show remarkable similarities in the kinematic properties of their interstellar medium gas: (i) first of all the blueshift of the ISM lines relative to the stars attributed to large-scale outflows, also observed in the large samples of $z \sim 2-3$ star-forming galaxies; (ii) the large broadness of the ISM lines with velocities spanning $\sim 1000 \mathrm{~km} \mathrm{~s}^{-1}$, from about -800 to $+300 \mathrm{~km} \mathrm{~s}^{-1}$ relative to the systemic redshift; and (iii) the similarity of the ISM line 


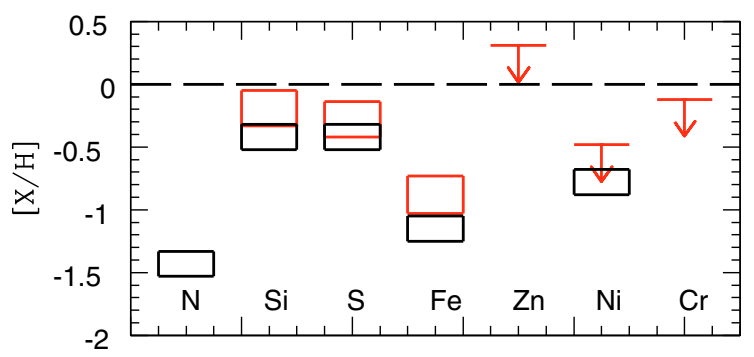

Fig. 10. Comparison of the elemental abundances, $[\mathrm{X} / \mathrm{H}]$, in the interstellar medium of the 8 o'clock arc (red) and MS 1512-cB58 (black). The height of the boxes reflects the $1 \sigma$ error on the abundance measurement, and the arrows refer to upper limits.

profiles among all ion species. As for the chemical abundances, the lensed LBGs seem also to present common characteristics. In Fig. 10 we compare the elemental abundances measured in cB58 and the 8 o'clock arc. They both show a very nice agreement between the $\alpha$-capture abundances, and a relatively high $\alpha$-enhancement relative to Fe-peak elements $(\sim 0.7$ dex $)$ which may be attributed to both dust depletion and/or nucleosynthesis. Galaxies associated with damped Ly $\alpha$ absorption line systems (DLAs) also offer the opportunity to study in detail the chemical abundances in the interstellar medium at high redshifts. The pattern of elemental abundances in LBGs appears to be different from DLAs, as already pointed out by Pettini et al. (2002). First, as for the metallicity, DLAs at all redshifts are generally metal-poor, the probability to find one DLA with a metallicity $Z>0.4 Z_{\odot}$ at $z=2-3$ is lower than $1 / 10$ (e.g., the recent compilation by Dessauges-Zavadsky et al. 2009). Second, the enhancement of $\alpha$-elements relative to Fe-peak elements due to nucleosynthetic effects seems very elusive to pin down in these galaxies (Prochaska \& Wolfe 2002; Dessauges-Zavadsky et al. 2006). These chemical differences suggest that star formation does not proceed in the same way in LBGs as in DLAs (Jimenez et al. 1999). There are also obvious differences in the kinematics of the ISM gas between LBGs and DLAs. The major one is the broadness of the ISM line profiles that span only $300 \mathrm{~km} \mathrm{~s}^{-1}$ in DLAs (e.g., Wolfe \& Prochaska 2000a,b). Furthermore, contrary to LBGs, in DLAs significant differences are observed between the profiles of low- and high-ionization lines. Given all these distinctions, DLAs very likely trace another galaxy population at high redshift than the LBGs.

\section{The He II emission}

The detection of the He II $\lambda 1640$ emission line in the 8 o'clock arc and in FOR J0332-3557 studied by Cabanac et al. (2008) betrays the presence of very massive stars - the WolfRayet stars - in these high-redshif galaxies. In nearby galaxies, this and other WR features are well known tracers of massive star-forming regions (Conti 1991; Schaerer \& Vacca 1998; Schaerer et al. 1999). In distant starbursts with high star formation rates, the timescale of their star formation activity is expected to be most likely relatively long compared to the lifetime of the massive stars ( $\$ 10 \mathrm{Myr})$. When star formation proceeds over long timescales, recently updated models predict a He II emission with equivalent widths $W_{0}$ (He II $) \sim-0.5 \AA$ for metallicities $\sim 0.4$ solar, and larger for higher metallicities (Brinchmann et al. 2008; Schaerer \& Vacca 1998). The observations of the few lensed LBGs appear in agreement with this prediction. Indeed, the 8 o'clock arc with its high metallicity, $Z \sim 0.8 Z_{\odot}$, shows a strong He II $\lambda 1640$ line. FOR J0332-3557 also shows a strong He II emission, although no accurate metallicity estimate is currently available for this LBG. In contrast, in cB58 and the Horseshoe, both characterized by lower metallicities, $Z \sim 0.5 Z_{\odot}$, the He II emission has not been detected, being quite likely below the detection threshold. Alternatively, differences in their star formation histories, age dependent extinction (Leitherer et al. 2002), or other effects could be invoked to explain the difference in strength of the He II line. Finally, it is also natural that $W_{0}(\mathrm{He}$ II) is larger in the 8 o'clock arc than in the composite spectrum of $z \sim 3$ LBGs, as our object is among the brightest, most massive, and most metal-rich LBGs.

\section{The Ly $\alpha$ profile}

The most striking difference between LBGs and also lensed LBGs observed so far is certainly the morphology of their Ly $\alpha$ profile. While in cB58, the 8 o'clock arc, FOR J0332-3557, and the Eye the $\operatorname{Ly} \alpha$ line is dominated by a damped absorption profile on top of which is superposed a weak emission (except in the Eye, where no emission is observed), in the Horseshoe the Ly $\alpha$ line is characterized by a strong double-peak emission profile. The common property of the emission component is its redshift relative to the ISM absorption lines, also observed in the large samples of $z \sim 2-3$ star-forming galaxies. With its broad Ly $\alpha$ absorption profile and the available detailed information, the 8 o'clock arc offers a new opportunity to test the scenario proposed by Schaerer \& Verhamme (2008) and Verhamme et al. (2008) to explain the Ly $\alpha$ absorption in LBGs and the diversity of other observed Ly $\alpha$ line profiles. In fact, it turns out that our radiation transfer models work better for the 8 o'clock arc than for cB58 analyzed previously, where we had to account for deviations from a spherical shell model (Schaerer \& Verhamme 2008). A homogeneous spherical shell model with a constant outflow velocity, determined by the observations, is able in the case of the 8 o'clock arc to reproduce the observed Ly $\alpha$ line profile, and the required dust content agrees well with the attenuation measured from the Balmer decrement. Furthermore, the assumption of homogeneity is reasonable in the 8 o'clock arc, since the low-ionization ISM absorption lines indicate a (nearly) complete coverage of the UV source. The results obtained from the fit of the Ly $\alpha$ line therefore fully support the scenario proposed earlier (Schaerer \& Verhamme 2008; Verhamme et al. 2008), where we showed that the diversity of Ly $\alpha$ line profiles in LBGs and Ly $\alpha$ emitters (LAEs), from absorption to emission, is mostly due to variations of H I column density and dust content. This scenario also naturally explains the main correlations observed between the Ly $\alpha$ emission and other properties of LBGs highlighted by Shapley et al. (2003). In particular, our detailed spectrum of the 8 o'clock arc and the fit of the Ly $\alpha$ line support the explanation for the observed correlation of the velocity shift between the Ly $\alpha$ emission and ISM lines, $\Delta v(\operatorname{Ly} \alpha-\operatorname{ISM})$, with $W_{0}(\operatorname{Ly} \alpha)$. The increase of $\Delta v(\operatorname{Ly} \alpha-$ ISM $)$ with increasing $\operatorname{Ly} \alpha$ absorption is mostly due to an increase of the H I column density, as nicely observed between the 8 o'clock arc and FOR J0332-3557 $7^{5}$, which implies that multiple scattering/radiation transfer effects become more important. Despite the relatively high values of $\Delta v(\operatorname{Ly} \alpha-\operatorname{ISM})$, the bulk outflow velocities remain relatively modest in galaxies with strong Ly $\alpha$ absorption. Measuring the ISM absorption

\footnotetext{
${ }^{5}$ In the 8 o'clock arc we measure $\Delta v(\operatorname{Ly} \alpha-\mathrm{ISM}) \sim 690 \mathrm{~km} \mathrm{~s}^{-1}$ for $\log N(\mathrm{H} \mathrm{I})=20.57$, while in FOR J0332-3557 we measure $\Delta v(\mathrm{Ly} \alpha-$ $\mathrm{ISM}) \sim 830 \mathrm{~km} \mathrm{~s}^{-1}$ for $\log N(\mathrm{HI})=21.40$.
} 
and the stellar photospheric absorption line redshifts remains the most reliable method to determine outflow velocities.

\section{UV covering factor and ISM geometry}

The UV covering factor of the cold ISM gas in LBGs has been determined for cB58, the Horseshoe, and the Eye. While in cB58 the blackness of the strongest interstellar absorption lines indicates a nearly complete coverage of the UV continuum by the ISM (Pettini et al. 2002), strong evidence for a patchy ISM with a coverage of only $\sim 60 \%$ and $70-85 \%$ of the UV continuum was found in the Horseshoe and the Eye, respectively (Quider et al. 2009a,b). Our data of the 8 o'clock arc show no evidence for a partial coverage, resembling again the case of $\mathrm{cB} 58$. What determines these differences and which case may be more general for LBGs remains to be determined. Independently of this question, it is clear that the partial UV coverage must also affect the Ly $\alpha$ line profile, leading preferentially to strong $\operatorname{Ly} \alpha$ emission, as discussed by Quider et al. (2009a). In the Cosmic Eye, the unusual presence of several, especially redshifted, components of cold gas, the large extinction (Smail et al. 2007), and the exceptionally high H I column density may very well explain the absence of any Ly $\alpha$ emission. If partial UV coverage was a common phenomenon, the unifying scenario explaining the diversity of Ly $\alpha$ line profiles in LBGs and LAEs with changes in H I column density and dust content (see above) could not be upheld. Clearly, further detailed studies on the ISM of high-redshift galaxies are needed to address this and other related questions thoroughly.

Acknowledgements. The good quality of the spectra obtained in the first nights of the instrument at the telescope is the result of the successful efforts of the X-shooter consortium team. More than 60 engineers, technicians, and astronomers worked over more than 5 years on the project in Denmark, France, Italy, the Netherlands, and at ESO. We recall here in representation of the whole team the co-PIs P. Kjaergaard-Rasmussen, F. Hammer, R. Pallavicini, L. Kaper, and S. Randich. R. Pallavicini, one of the strongest supporter of the project, died prematurely just after the first light of the instrument. Special thanks go also to the ESO Commissioning team led by H. Dekker and including among others J. Lizon, R. Castillo, M. Downing, G. Finger, G. Fischer, C. Lucuix, E. Mason, and P. Santin. We thank the anonymous referee for a helpful and constructive report, and we are also grateful to M. Pettini for his useful comments. M.D.Z. and D.S. are supported by the Swiss National Science Foundation.

\section{References}

Adelberger, K. L., Steidel, C. C., Shapley, A. E., \& Petttini, M. 2003, ApJ, 584, 45

Allam, S. S., Tucker, D. L., Lin, H., et al. 2007, ApJ, 662, L51

Aloisi, A., Savaglio, S., Heckman, T. M., et al. 2003, ApJ, 595, 760

Belokurov, V., Evans, N. W., Moiseev, A., et al. 2007, ApJ, 671, L9

Brinchmann, J., Pettini, M., \& Charlot, S. 2008, MNRAS, 385, 769

Cabanac, R. A., Valls-Gabaud, D., Jaunsen, A. O., Lidman, C., \& Jerjen, H. 2005, A\&A, 436, L21

Cabanac, R. A., Valls-Gabaud, D., \& Lidman, C. 2008, MNRAS, 386, 2065

Chandar, R., Leitherer, C., \& Tremonti, C. A. 2004, ApJ, 604, 153

Coppin, K. E. K., Swinbank, A. M., Neri, R., et al. 2007, ApJ, 665, 936

Conti, P. S. 1991, ApJ, 377, 115

Crowther, P. A., Prinja, R. K., Pettini, M., \& Steidel, C. C. 2006, MNRAS, 368, 895

Dessauges-Zavadsky, M., Prochaska, J. X., D’Odorico, S., Calura, F., \& Matteucci, F. 2006, A\&A, 455, 93
Dessauges-Zavadsky, M., Ellison, S. L., \& Murphy, M. T. 2009, MNRAS, 396, L61

Diehl, H. T., Allam, S. S., Annis, J., et al. 2009, ApJ, 707, 686

Dijkstra, M., \& Loeb, A. 2008, MNRAS, 386, 492

Dijkstra, M., Haiman, Z., \& Spaans, M. 2006, ApJ, 649, 14

D’Odorico, S., Dekker, H., Mazzoleni, R., et al. 2006, SPIE, 6269, 98

Ellingson, E., Yee, H. K. C., Bechtold, J., \& Elston, R. 1996, ApJ, 466, L71

Erb, D. K., Steidel, C. C., Shapley, A. E., et al. 2006a, ApJ, 647, 128

Erb, D. K., Shapley, A. E., Pettini, M., et al. 2006b, ApJ, 644, 813

Finkelstein, S. L., Papovich, C., Rudnick, G., et al. 2009, ApJ, 700, 376

Fontana, A., \& Ballester, P. 1995, The Messenger, 80, 37

Giavalisco, M., Steidel, C. C., Adelberger, K. L., et al. 1998, ApJ, 503, 543

Goldoni, P., Royer, F., François, P., et al. 2006, SPIE, 6269, 80

Grevesse, N., Asplund, M., \& Sauval, A. J. 2007, Space Sci. Rev., 130, 105

Hainline, K. N., Shapley, A. E., Kornei, K. A., et al. 2009, ApJ, 701, 52

Heckman, T. K., Lehnert, M. D., Strickland, D. K., \& Armus, L. 2000, ApJS, 129,493

Jenkins, E. B., \& Tripp, T. M. 2006, ApJ, 637, 548

Jimenez, R., Bowen, D. V., \& Matteucci, F. 1999, ApJ, 514, L83

Kelson, D. D. 2003, PASP, 115, 688

Kubo, J. M., Allam, S. S., Annis, J., et al. 2009, ApJ, 696, L61

Law, D. R., Steidel, C. C., Erb, D. K., et al. 2007, ApJ, 656, 1

Lebouteiller, V., Kunth, D., Thuan, T. X., \& Désert, J. M. 2009, A\&A, 494, 915

Leitherer, C., Schaerer, D., Goldader, J. D., et al. 1999, ApJS, 123, 3

Leitherer, C., Leão, J. R. S., Heckman, T. M., et al. 2001, ApJ, 550, 724

Leitherer, C., Calzetti, D., \& Martins, L. P. 2002, ApJ, 574, 114

Lin, H., Buckley-Geer, E., Allam, S. S., et al. 2009, ApJ, 699, 1242

Maiolino, R., Nagao, T., Grazian, A., et al. 2008, A\&A, 488, 463

Morton, D. C. 2003, ApJS, 149, 205

Noterdaeme, P., Petitjean, P., Ledoux, C., \& Srianand, R. 2009, A\&A, 505, 1087

Pettini, M., \& Pagel, B. E. J. 2004, MNRAS, 348, L59

Pettini, M., Ellison, S. L., Steidel, C. C., \& Bowen, D. V. 1999, ApJ, 510, 576

Pettini, M., Steidel, C. C., Adelberger, K. L., Dickinson, M., \& Giavalisco, M. 2000, ApJ, 528, 96

Pettini, M., Shapley, A. E., Steidel, C. C., et al. 2001, ApJ, 554, 981

Pettini, M., Rix, S. A., Steidel, C. C., et al. 2002, ApJ, 569, 742

Prochaska, J. X., \& Wolfe, A. M. 2002, ApJ, 566, 68

Prochaska, J. X., Herbert-Fort, S., \& Wolfe, A. M. 2005, ApJ, 635, 123

Quider, A. M., Pettini, M., Shapley, A. E., \& Steidel, C. C. 2009a, MNRAS, 398, 1263

Quider, A. M., Shapley, A. E., Pettini, M., Steidel, C. C., \& Stark, D. P. 2009b, MNRAS, in press [arXiv: 0910.0840]

Reddy, N. A., Steidel, C. C., Pettini, M., et al. 2008, ApJS, 175, 48

Rix, S. A., Pettini, M., Leitherer, C., Bresolin, F., \& Kudritzki, R.-P. 2004, ApJ, 615,98

Savage, B. D., \& Sembach, K. R. 1991, ApJ, 379, 245

Savage, B. D., \& Sembach, K. R. 1996, ARA\&A, 34, 279

Savaglio, S., Panagia, N., \& Padovani, P. 2002, ApJ, 567, 702

Schaerer, D. 2003, A\&A, 397, 527

Schaerer, D., \& Vacca, W. D. 1998, ApJ, 497, 618

Schaerer, D., \& Verhamme, A. 2008, A\&A, 480, 369

Schaerer, D., Contini, T., \& Pindao, M. 1999, A\&AS, 136, 35

Shapley, A. E., Steidel, C. C., Adelberger, K. L., et al. 2001, ApJ, 562, 95

Shapley, A. E., Steidel, C. C., Pettini, M., \& Adelberger, K. L. 2003, ApJ, 588, 65

Shapley, A. E., Erb, D. K., Pettini, M., Steidel, C. C., \& Adelberger, K. L. 2004, ApJ, 612, 108

Smail, I., Swinbank, A. M., Richard, J., et al. 2007, ApJ, 654, L33

Stark, D. P., Swinbank, A. M., Ellis, R. S., et al. 2008, Nature, 455, 775

Steidel, C. C., Giavalisco, M., Pettini, M., Dickinson, M., \& Adelberger, K. L. 1996, ApJ, 462, L17

Stenflo, J. O. 1980, A\&A, 84, 68

Teplitz, H. I., McLean, I. S., Becklin, E. E., et al. 2000, ApJ, 533, L65

Verhamme, A., Schaerer, D., \& Maselli, A. 2006, A\&A, 460, 397

Verhamme, A., Schaerer, D., Atek, H., \& Tapken, C. 2008, A\&A, 491, 89

Witt, A. N., \& Gordon, K. D. 2000, ApJ, 528, 79

Wolfe, A. M., \& Prochaska, J. X. 2000a, ApJ, 545, 591

Wolfe, A. M., \& Prochaska, J. X. 2000b, ApJ, 545, 603

Zheng, Z., \& Miralda-Escudé, J. 2002, ApJ, 578, 33 